\title{
Limit cycles via higher order perturbations for some piecewise differential systems
}

\author{
Claudio A. Buzzi ${ }^{\text {a }}$, Maurício Firmino Silva Lima ${ }^{\text {b }}$, Joan Torregrosa ${ }^{\text {c,* }}$ \\ a Departamento de Matemática, Universidade Estadual Paulista, São José do Rio Preto, Brazil \\ b Centro de Matemática Computação e Cognição. Universidade Federal do ABC, 09210-170. Santo Andre. S.P., Brazil \\ c Departament de Matemàtiques, Universitat Autònoma de Barcelona, 08193 Bellaterra, Barcelona, Spain
}

\section{H I G H L I G H T S}

- The number of limit cycles bifurcating from linear centers.

- Piecewise perturbations in two zones with a straight line of separation.

- Upper bound for that number for arbitrary perturbation order.

- The upper bounds are reached for perturbations of order one and two.

- Some applications to concrete families.

\section{A R T I C L E I N F O}

\section{Article history:}

Received 21 August 2017

Received in revised form 20 December 2017

Accepted 15 January 2018

Available online 31 January 2018

Communicated by J. Dawes

\section{Keywords:}

Non-smooth differential system

Limit cycle in Melnikov higher order

perturbation

Liénard piecewise differential system

\begin{abstract}
A B S T R A C T
A classical perturbation problem is the polynomial perturbation of the harmonic oscillator, $\left(x^{\prime}, y^{\prime}\right)=$ $(-y+\varepsilon f(x, y, \varepsilon), x+\varepsilon g(x, y, \varepsilon))$. In this paper we study the limit cycles that bifurcate from the period annulus via piecewise polynomial perturbations in two zones separated by a straight line. We prove that, for polynomial perturbations of degree $n$, no more than $N n-1$ limit cycles appear up to a study of order $N$. We also show that this upper bound is reached for orders one and two. Moreover, we study this problem in some classes of piecewise Liénard differential systems providing better upper bounds for higher order perturbation in $\varepsilon$, showing also when they are reached. The Poincaré-Pontryagin-Melnikov theory is the main technique used to prove all the results.
\end{abstract}

(c) 2018 Elsevier B.V. All rights reserved.

\section{Introduction}

In last decades, piecewise differential systems have been useful for modeling real processes and different modern devices. For simplicity also the linear piecewise differential systems provide adequate models with very accurate results close to the observed data. See for more details [1,2]. Although in recent years these systems have attracted a good deal of attention, the first stages of modeling with piecewise systems started with Andronov and coworkers, see [3].

In this paper, we study the number of isolated periodic orbits, the so called limit cycles, of a polynomial piecewise perturbation of degree $n$ of a linear center, when the separation curve is a straight line $\Sigma$ passing through the center. That is, we consider systems

\footnotetext{
* Corresponding author.

E-mail addresses: buzzi@ibilce.unesp.br (C.A. Buzzi), mauricio.lima@ufabc.edu.br (M.F.S. Lima), torre@mat.uab.cat (J. Torregrosa).
}

written as

$\left(x^{\prime}, y^{\prime}\right)=\left(-\frac{\partial H}{\partial y}+\sum_{i=1}^{N} \varepsilon^{i} f_{i}^{ \pm}(x, y), \frac{\partial H}{\partial x}+\sum_{i=1}^{N} \varepsilon^{i} g_{i}^{ \pm}(x, y)\right)$

such that the unperturbed system has a center at $\left(x_{c}, y_{c}\right)$ with $H$ a quadratic polynomial and the perturbations $f_{i}^{ \pm}$and $g_{i}^{ \pm}$are polynomials of degree $n$ defined in each side of $\Sigma$. Via an affine change of coordinates, if necessary, it is not restrictive to assume that we are studying the piecewise polynomial perturbation of the harmonic oscillator. Consequently, we consider the above system in the form

$\left(x^{\prime}, y^{\prime}\right)=\left(-y+\sum_{i=1}^{N} \varepsilon^{i} f_{i}^{ \pm}(x, y), x+\sum_{i=1}^{N} \varepsilon^{i} g_{i}^{ \pm}(x, y)\right)$,

with $f_{i}^{ \pm}$and $g_{i}^{ \pm}$polynomials of degree $n$, defined in $\Sigma_{0}^{ \pm}=\{(x, y) \in$ $\left.\mathbb{R}^{2}: \pm y \geq 0\right\}$. This problem, for non piecewise perturbations, was studied by Iliev in [4], proving that the number of limit cycles is 
bounded by $[N(n-1) / 2]$. In this paper, we prove by induction on the degree $n$ that, in the piecewise case, the upper bound is approximately doubled. In the full paper [.] denotes the integer part function.

The main tool used in this paper will be the PoincaréPontryagin-Melnikov technique. In this theory the limit cycles bifurcate from the level sets of a Hamiltonian $H$. More concretely, from the closed level sets that do not have equilibrium points. For system (2), the limit cycles $\gamma_{\varepsilon}(r)$ bifurcate from the level sets $\varphi_{r}=\left\{x^{2}+y^{2}=r^{2}\right\}$. That is, $\gamma_{\varepsilon}(r)$ tends to $\varphi_{r}$ when $\varepsilon$ goes to 0 . Here, this type of periodic orbits, with $r>0$ and when they are isolated, are called limit cycles bifurcating from the period annulus of the center. It is clear that, for $\varepsilon$ small enough, this type of solutions of (2) are well defined in the complement set of any neighborhood of the origin. We can say that these periodic orbits are away from the origin. Then, the Filippov convention is not necessary here, see [2]. In fact, there are other mechanism, like averaging method, to study the limit cycles bifurcating from the closed level sets of a Hamiltonian. But, in the plane and for an autonomous perturbation, they are equivalent, see [5] or [6]. In Section 2 we recall the necessary results for proving our main goals.

Next result provides upper bounds for the number of limit cycles bifurcating from the period annulus of the harmonic oscillator with piecewise polynomials of degree $n$.

Theorem 1.1. The maximum number of limit cycles of system (1) bifurcating from the unperturbed period annulus is at most $n$ up to order 1 and at most $N n-1$ up to order $N \geq 2, n \geq 1$. Moreover, there exist perturbation parameters such that for orders 1 and 2 the upper bounds are reached.

The Poincaré-Pontryagin-Melnikov technique provides a function, $M_{N}$, for each perturbation order and the isolated periodic orbits are given from the simple zeros of it. The study of the number of zeros cannot be done in general because of the difficulties to find explicitly the functions $M_{N}$. There are few papers dealing with these objectives, see for example $[4,7,8]$. The upper bounds for the number of limit cycles up to order $N$ are given by the number of simple zeros of $M_{N}$ and, from the upper bound presented in the above result, it seems that this number always increases with $N$. This is not the case for degree $n=1$ perturbations in piecewise continuous and piecewise sewing, see [9] and [10], respectively. The study up to order seven and for general piecewise perturbation of degree 1 is done in [11], where it is proved that the increasing sequence of zeros is $1,1,2,3,3,3,3$. Quadratic and cubic perturbations up to order 5 are studied in [12], where the authors prove that there exist polynomial perturbations such that $M_{N}$ has 2, 3, 5 , 6 , and 8 simple zeros for $n=2$ and 3, 5, 8, 11, and 13 for $n=3$. Another example exhibiting 3 limit cycles for the quadratic family is given in [13].

It is clear that the study about how the number of limit cycles increases with the perturbation order is far to be solved, even in the case of small degrees. Only for some special families this problem can be solved. For example, if we consider the non-piecewise perturbation of the harmonic oscillator in the Liénard family,

$\left(x^{\prime}, y^{\prime}\right)=\left(-y+\sum_{i=1}^{N} \varepsilon^{i} f_{i}(x), x\right)$,

with $f_{i}$ polynomials of degree $n$. In [8] it is proved that all the functions $M_{N}$ has the same number, [ $\left.(n-1) / 2\right]$, of simple zeros as $M_{1}$. For the classical Liénard family $\left(x^{\prime}, y^{\prime}\right)=(-y+f(x), x)$, Lins, de Melo and Pugh conjectured in [14] that the maximal number of limit cycles is exactly [ $n-1) / 2]$. Zuppa in [15] proved this conjecture for the limit cycles bifurcating from the origin via a degenerate Hopf bifurcation. The conjecture is also true for degree $n=4$, see [16], but it fails for higher degrees, see [17] and [18].
As in the smooth case, the upper bounds will not be reached in general. But this is a question not solved yet even for the smooth case. The general study of the zeros of the Melnikov functions up to every order is also very difficult to be done for a polynomial family of degree $n$. So, it is quite natural to restrict our attention to study only some special families under piecewise perturbation. In what follows, we present some results about the number of limit cycles in some piecewise Liénard families. This kind of problems were also treated in [19] using Lyapunov quantities and in [20] for rational Liénard systems. Recently, Sheng in [21] uses Melnikov functions to study also the number of limit cycles for other piecewise Liénard families.

In this work line we present our main results. First, in Theorem 1.2, we deal with a Liénard family where the upper bounds given in the above theorem are decreased and achieved for perturbations of first and second orders. Next, adding some symmetry hypothesis, we can prove, in Theorem 1.3, that no limit cycles appear or the number of limit cycles up to any order of perturbation coincides with the ones bifurcating from the first order. Finally, changing the discontinuity line, from the $x$-axis to the $y$-axis, in Theorem 1.4, we prove that the Melnikov functions of any order have the same aspect and the upper bound is reached always. In this case we have proved that the number of limit cycles does not increase with the order of perturbation.

Consider now,

$\left(x^{\prime}, y^{\prime}\right)=\left(-y+\sum_{i=1}^{N} \varepsilon^{i} f_{i}^{ \pm}(x), x\right)$,

defined in $\Sigma_{0}^{ \pm}=\left\{(x, y) \in \mathbb{R}^{2}: \pm y \geq 0\right\}$, where $f_{i}^{ \pm}$are polynomials of degree $n$.

Theorem 1.2. The maximum number of limit cycles of system (3) bifurcating from the unperturbed period annulus is at most $[(n-1) / 2]$ and $n+[(n-1) / 2]$ for orders 1 and 2, respectively. Moreover, there exist polynomials $f_{i}^{ \pm}$such that for orders 1 and 2 these upper bounds are reached.

\section{Theorem 1.3.}

(a) When $f_{i}^{ \pm}$are even polynomials, then system (3) has a center at the origin for every $\varepsilon$.

(b) When $f_{i}^{ \pm}$are odd polynomials, the maximum number of limit cycles of system (3) bifurcating from the period annulus is at most $(n-1) / 2$ up to any order of perturbation. Moreover, there exist polynomials $f_{i}^{ \pm}$such that this upper bound is reached.

Last result deals with system (3) but changing the separation line to the $y$-axis. In this case, we consider the system

$\left(x^{\prime}, y^{\prime}\right)=\left(-y+\sum_{i=1}^{N} \varepsilon^{i} f_{i}^{ \pm}(x), x\right)$,

defined in $\Sigma_{1}^{ \pm}=\left\{(x, y) \in \mathbb{R}^{2}: \pm x \geq 0\right\}$, where $f_{i}^{ \pm}$are polynomials of degree $n$.

Theorem 1.4. The maximum number of limit cycles of family (4) bifurcating from the period annulus is at most $n$ up to any order of perturbation. Moreover, there exist polynomials $f_{i}^{ \pm}$such that the upper bound is reached.

The paper is organized as follows. In Section 2 we introduce the main tools for proving all the results. Section 3 is devoted to the proof of Theorem 1.1, first proving the upper bounds and second providing explicit examples exhibiting that number of limit cycles. The upper bounds are proved by induction on the degree of the perturbation. Sections 4 and 5 deal with the proofs of 
Theorems 1.2, 1.3, and 1.4. Finally, in Section 6 we study higher order perturbations for some fixed families, but for small values of $n$. First, we show that the number of limit cycles provided by Theorem 1.2 does not increase up to order 4 . This is done in Proposition 6.1. Second, for a generalized Liénard family and up to order 5 , we show that the number of limit cycles increases with the perturbation order but not as Theorem 1.1 says. This is done in Proposition 6.2. The conclusions of these results suggest that, when we fix the degree of the polynomial perturbation, the number of limit cycles will increase with the order of the perturbation up to a maximal number. We can say that there is a saturation in the growth of the number of limit cycles. This phenomenon can be observed in piecewise linear systems with a straight line of separation (see [11]), when the separation is non-regular (see [22]), and also in analytical families (see [8]).

\section{The return map and the Poincaré-Pontryagin-Melnikov functions}

This section is devoted to present the main tools that we need to state and prove the results of this paper. We closely follow the presentation in [23], which decomposes an arbitrary one-form in polar coordinates. It is based on the decompositions introduced by Françoise in [24-26]. This procedure is also used in [11], but only for piecewise linear differential systems.

The vector field $X$ given in (1) with the separation line $\Sigma_{0}=$ $\{(x, 0): x \in \mathbb{R}\}$, in polar coordinates $(x, y)=(r \cos \theta, r \sin \theta)$, can be written as

$$
(\dot{r}, \dot{\theta})= \begin{cases}\left(\sum_{i=1}^{N} \varepsilon^{i} R_{i}^{+}(r, \theta),\right. & \\ \left.1+\sum_{i=1}^{N} \varepsilon^{i} \Theta_{i}^{+}(r, \theta)\right) & \text { if } \theta \in[0, \pi), \\ \left(\sum_{i=1}^{N} \varepsilon^{i} R_{i}^{-}(r, \theta),\right. & \text { if } \theta \in[\pi, 2 \pi),\end{cases}
$$

where $R_{i}^{ \pm}, \Theta_{i}^{ \pm}$are analytic functions in $r, \sin \theta$ and $\cos \theta$. The above system can also be expressed as

$$
\begin{cases}d H+\sum_{i=1}^{N} \varepsilon^{i} \omega_{i}^{+}=0 & \text { if } \theta \in[0, \pi), \\ d H+\sum_{i=1}^{N} \varepsilon^{i} \omega_{i}^{-}=0 & \text { if } \theta \in[\pi, 2 \pi),\end{cases}
$$

where $H(r)=\left(x^{2}+y^{2}\right) / 2=r^{2} / 2$, and $\omega_{i}^{ \pm}=\omega_{i}^{ \pm}(r, \theta)$ are analytic one-forms, $2 \pi$-periodic in $\theta$ and polynomial in $r$.

We denote by $X^{ \pm}$each vector field (5) defined in $\Sigma_{0}^{ \pm}=\{(x, y) \in$ $\left.\mathbb{R}^{2}: \pm y \geq 0\right\}$. Clearly, we need to define $X$ in the separation line $\Sigma_{0}$. We say that a solution of the above vector field, $X^{ \pm}$, is of sewing type if when it crosses the separation line, $\Sigma_{0}$, satisfies that $X^{+} \cdot(0,1)$ and $X^{-} \cdot(0,1)$ have the same sign in the intersection points with $\Sigma_{0}$. When this is not the case we can use the Filippov convention, see [2], to define the vector field over such special points in $\Sigma_{0}$. These points define the so called sliding set. In this paper we are dealing with isolated periodic orbits bifurcating from a center. So, by continuity, we are interested only in the so called limit cycles of sewing type. The periodic orbits that cross the sliding set will be studied in future works. This is the reason why we do not detail more such special solutions.

We define straightaway the main tool used in this paper: the Poincaré map. Let $r^{+}(\theta, \rho)$ (resp. $\left.r^{-}(\theta, \rho)\right)$ be the solution of $X$ such

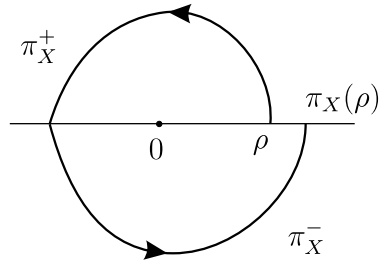

(a)

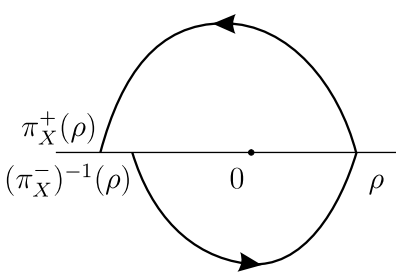

(b)
Fig. 1. (a) Return map and (b) half-return maps $\pi_{X}^{+}$and $\left(\pi_{X}^{-}\right)^{-1}=\pi_{\mathcal{R}(X)}^{+}$of system (6).

that $r^{+}(0, \rho)=\rho$ (resp. $\left.r^{-}(\pi, \rho)=\rho\right)$. Then, we define the positive Poincaré half-return map as $\pi_{X}^{+}(\rho)=r^{+}(\pi, \rho)$ and the negative Poincaré half-return map as $\pi_{X}^{-}(\rho)=r^{-}(2 \pi, \rho)$. The complete Poincaré return map associated to $X$ is given by the composition of these two maps

$\pi_{X}(\rho)=\pi_{X}^{-}\left(\pi_{X}^{+}(\rho)\right)$,

see Fig. 1(a). We can also write them, for system (5), as a power series in $\varepsilon$ as

$\pi_{X}^{ \pm}(\rho, \varepsilon)=\rho+\sum_{i=1}^{N} \varepsilon^{i} p_{i}^{ \pm}(\rho), \quad$ and $\quad \pi_{X}(\rho, \varepsilon)=\rho+\sum_{i=1}^{N} \varepsilon^{i} p_{i}(\rho)$.

It is important to mention that both maps, $\pi_{X}^{+}$and $\pi_{X}^{-}$, are analytic in $\rho$ and $\varepsilon$, so the complete Poincaré map associated to $X$ is also analytic.

The result below relates the half-return map on $\Sigma_{0}^{-}$of the vector field $X$ with the half-return map on $\Sigma_{0}^{+}$of the transformed vector field $\mathcal{R}(x)$ which is defined by the reversibility property. That is, for each solution $\gamma(t)=(x(t), y(t))$ of $X$ then $\mathcal{R}(\gamma(t))=$ $(x(-t),-y(-t))$ is a solution of $\mathcal{R}(X)$. The proof follows directly from the reversibility property. Moreover the study of the number of zeros of the displacement map, $\pi_{X}(\rho)-\rho$, is easier.

Lemma 2.1. The first non-zero term of the map $\pi_{X}(\rho)-\rho$ coincides with the first non-zero term of the map

$\pi_{X}^{+}(\rho)-\left(\pi_{X}^{-}\right)^{-1}(\rho)=\pi_{X}^{+}(\rho)-\pi_{\mathcal{R}(X)}^{+}(\rho)$,

where $\mathcal{R}(X)=(-P(x,-y), Q(x,-y))$ for $X=(P, Q)$. See Fig. $1(b)$.

From the above result we can study the general expression for $r^{+}$in the region $\Sigma_{0}^{+}$and then, using the reversibility property, we can get $r^{-}$from $r^{+}$. In other words, we only need to consider $\theta \in[0, \pi)$.

Now, we recall the decomposition of a one-form given in [24] and the extension to piecewise differential forms, in polar coordinates, in [23] to the computation of the Poincaré map. The initial value problem

$$
\begin{aligned}
& \left(x^{\prime}, y^{\prime}\right)=(-y+\varepsilon P(x, y, \varepsilon), x+\varepsilon Q(x, y, \varepsilon)), \\
& (x(0, \varepsilon), y(0, \varepsilon))=(\rho, 0),
\end{aligned}
$$

where $P$ and $Q$ are smooth functions, can be written, using usual polar coordinates $(x, y)=(r \cos \theta, r \sin \theta)$, as

$$
\left\{\begin{array}{l}
d H+\sum_{i=1}^{N} \varepsilon^{i} \omega_{i}=0, \\
r(0, \varepsilon, \rho)=\rho .
\end{array}\right.
$$

Here $H(r)=r^{2} / 2$ and $\omega_{i}=\omega_{i}(r, \theta)$ are smooth one-forms $2 \pi$-periodic in $\theta$. The solution $r(\theta, \varepsilon, \rho)$ of $(7)$ writes as $r(\theta, \varepsilon, \rho)=$ $\sum_{i=0}^{N} r_{i}(\theta, \rho) \varepsilon^{i}$ and it can be easily checked that $r(\theta, 0, \rho)=$ $r_{0}(\theta, \rho) \equiv \rho$. See Fig. 2 .

The next result follows straightforward from the decompositions of [24-26]. 


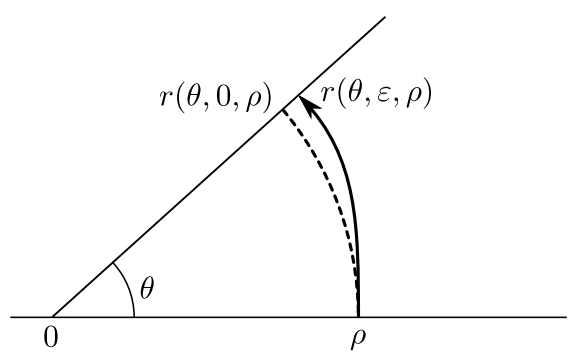

Fig. 2. Solution of Eq. (7) for $\varepsilon$ small enough.

Lemma 2.2. Let $\Omega=\alpha(r, \theta) d r+\beta(r, \theta) d \theta$ be an arbitrary analytic one-form, $2 \pi$-periodic in $\theta$ and $H(r)=r^{2} / 2$. Then there exist functions $h(r, \theta), S(r, \theta)$ and $F(r)$ also $2 \pi$-periodic in $\theta$ and defined by $F(r)=\frac{1}{2 \pi} \int_{0}^{2 \pi} \beta(r, \psi) d \psi, S(r, \theta)=\int_{0}^{\theta} \beta(r, \psi) d \psi-F(r) \theta$ and $h(r, \theta)=\left(\alpha(r, \theta)-\frac{\partial S(r, \theta)}{\partial r}\right) / H^{\prime}(r)$, such that $\Omega=\Omega^{0}+\Omega^{1}$ where $\Omega^{0}=h d H+d S, \Omega^{1}=F(r) d \theta$, and $\int_{H=\rho^{2} / 2} \Omega^{0}=0$, $\int_{H=\rho^{2} / 2} \Omega^{1}=\int_{H=\rho^{2} / 2} \Omega$.

Next proposition allows us to obtain $r_{i}(\theta, \rho)$ for every $i$ from the decomposition of a one-form given in lemma behind, see [23]. Although it is a direct consequence of the results in [23], we have included it here by completeness and because this expression for the function $r_{i}$ is more explicit and clear than that of the aforementioned reference.

Proposition 2.3. Let $r(\theta, \varepsilon, \rho)=\sum_{i=0}^{N} r_{i}(\theta, \rho) \varepsilon^{i}$ be the solution of (7). Assume that, from the decomposition given in Lemma 2.2, the functions $F_{i}(r), h_{i}(r, \theta)$, and $S_{i}(r, \theta)$ are defined inductively as $h_{0}=1$ and $-\Omega_{i}:=-\sum_{j=1}^{i} \omega_{j} h_{i-j}=h_{i} d H+d S_{i}+F_{i} d \theta$, for $i=1, \ldots, N$. If the functions $r_{0}(\theta, \rho)=\rho$, and $r_{i}(\theta, \rho)$, for $i=1, \ldots, N-1$ are known, then

$$
\begin{aligned}
\rho r_{N}(\theta, \rho)= & -\frac{1}{2} \sum_{i=1}^{N-1} r_{i}(\theta, \rho) r_{N-i}(\theta, \rho)+\mathcal{G}(S)(\rho, \theta) \\
& +\int_{0}^{\theta} \mathcal{G}(F)(\rho, \psi) d \psi,
\end{aligned}
$$

where $S=\left(S_{1}, \ldots, S_{N}\right)$ and $F=\left(F_{1}, \ldots, F_{N}\right)$. The operator $\mathcal{G}$ on $f=\left(f_{1}, \ldots, f_{N}\right)$ is defined by

$$
\begin{aligned}
\mathcal{G}(f)(\rho, \theta)= & f_{N}(\rho, \theta)+\sum_{i=1}^{N-1} \sum_{j=1}^{N-i} D_{1}^{j}\left(f_{i}\right)(\rho, \theta) \\
& \times \sum_{\substack{m_{1}+2 m_{2}+\cdots+\ell m_{\ell}=N-i \\
m_{1}+m_{2}+\cdots+m_{\ell}=j}} r_{1}^{m_{1}}(\theta, \rho) r_{2}^{m_{2}}(\theta, \rho) \cdots r_{\ell}^{m_{\ell}}(\theta, \rho)
\end{aligned}
$$

with $D_{1}^{j}(g)(\rho, \theta)=\left.\frac{\partial^{j} g(r, \theta)}{\partial r^{j}}\right|_{r=\rho}$.

The above result applied to the computation of the first terms in the Taylor series in $\varepsilon$ of $r(\theta, \rho, \varepsilon)$ gives

$$
\begin{aligned}
\rho r_{1}(\theta, \rho)= & S_{1}(\rho, \theta)+F_{1}(\rho) \theta, \\
\rho r_{2}(\theta, \rho)= & -\frac{1}{2} r_{1}(\theta, \rho)^{2}+S_{2}(\rho, \theta)+D_{1}\left(S_{1}\right)(\rho, \theta) r_{1}(\theta, \rho) \\
& +F_{2}(\rho) \theta+F_{1}^{\prime}(\rho) \int_{0}^{\theta} r_{1}(\psi, \rho) d \psi,
\end{aligned}
$$

with $D_{1}\left(S_{1}\right)(\rho, \theta)=\left.\frac{\partial S_{1}(r, \theta)}{\partial r}\right|_{r=\rho}$.

Proof of Proposition 2.3. From Theorem 2.4 in [23] and all the notation of the statement we have that for any $N \in \mathbb{N}, r(\theta, \varepsilon, \rho)$ satisfies the following implicit equation

$$
\begin{aligned}
\frac{r^{2}(\theta, \varepsilon, \rho)-\rho^{2}}{2}= & \sum_{i=1}^{N} \varepsilon^{i}\left(\int_{0}^{\theta} F_{i}(r(\psi, \varepsilon, \rho)) d \psi\right. \\
& \left.+\left.S_{i}(r(\psi, \varepsilon, \rho), \psi)\right|_{\psi=0} ^{\psi=\theta} \begin{array}{l}
\psi=0 \\
\psi=
\end{array}\right)+\mathcal{O}_{\varepsilon^{N+1}}
\end{aligned}
$$

First we substitute $r(\theta, \varepsilon, \rho)=\sum_{i=0}^{N} r_{i}(\theta, \rho) \varepsilon^{i}$ in the above equation. The proof follows, by induction, equating the coefficient of $\varepsilon^{n}$ in both sides of Eq. (9).

Remark 2.4. The functions $h$ and $S$ given in Lemma 2.2 are not unique. In fact, for a fixed pair $h, S$ such that $\Omega=h d H+d S+F(r) d \theta$ we have $\Omega=\widetilde{h} d H+d \widetilde{S}+F(r) d \theta$ with $\widetilde{h}=h-f^{\prime}(r) / r$ and $\widetilde{S}=S+f(r)$ for any function $f(r)$. The new decomposition satisfies also that $\widetilde{h}, \widetilde{S}$ are $2 \pi$-periodic in $\theta$. In particular, the conclusions of the above proposition does not depend on which decomposition is used.

Using the notions and results introduced until now, we can state the main result used in the next sections, the so called PoincaréPontryagin-Melnikov functions. The solution of system (6) in power series in $\varepsilon$ writes as $r^{ \pm}(\theta, \varepsilon, \rho)=\sum_{i=0}^{N} r_{i}^{ \pm}(\theta, \rho) \varepsilon^{i}$, and it can be easily checked that $r^{ \pm}(\theta, 0, \rho)=r_{0}^{ \pm}(\theta, \rho) \equiv \rho$. Lemma 2.1 and Proposition 2.3 allow us to compute $r_{i}^{ \pm}(\theta, \rho)$ for every value of $\theta$. Consequently the first non-vanishing term of the complete return map far from the sliding segment is obtained from the next result.

Proposition 2.5. Denoting by $\widehat{M}_{N}(\rho)=r_{N}^{+}(\pi, \rho)-r_{N}^{-}(-\pi, \rho)$, the Poincaré-Pontryagin-Melnikov functions, or Melnikov functions for shortness, of order $N$, for system (5), are given by

$$
\begin{aligned}
& M_{1}(\rho)=\widehat{M}_{1}(\rho) \text { and } M_{N}(\rho)=\left.\widehat{M}_{N}(\rho)\right|_{\left\{M_{k}(\rho) \equiv 0, k=1, \ldots, N-1\right\}}, \\
& \quad \text { for } N \geq 2 \text {. }
\end{aligned}
$$

Moreover, for $\varepsilon$ small enough, each simple zero, $\hat{\rho}$, of $M_{N}(\rho)$ gets a limit cycle of system (5). The limit cycle bifurcates from the level curve $x^{2}+y^{2}=\hat{\rho}^{2}$.

We remark that the above proposition provides the functions $\widehat{M}_{k}(\rho)$, in terms of the coefficients of the perturbations, for every order $k$, but only coincides with the Melnikov function of a fixed order $N$ when the previous ones vanish identically.

This section ends with two technical results that they will be useful for the explicit computations in the next sections.

Lemma 2.6. The next properties hold.

(a) $s_{k}:=\frac{1}{2 \pi} \int_{0}^{2 \pi} \sin ^{k} \psi d \psi=\frac{(k-1) ! !}{k ! !} \hat{\delta}_{k}$ where $\hat{\delta}_{k}= \begin{cases}1, & \text { if } k \text { is even } \\ 0, & \text { if } k \text { is odd. }\end{cases}$

(b) $c_{k}:=\frac{1}{2 \pi} \int_{0}^{2 \pi} \cos ^{k} \psi d \psi=s_{k}$.

(c) $\mathcal{S}_{k}(\theta):=\int_{0}^{\theta} \sin ^{k} \psi d \psi-s_{k} \theta$ has the form $p_{k}(\cos \theta)$ if $k$ odd or $p_{k-1}(\cos \theta) \sin \theta$ if $k$ even, where $p_{j}$ is a polynomial of degree $j$ in $\cos \theta$. In particular, $\mathcal{S}_{k}(\theta)$ is $2 \pi$-periodic.

(d) $\mathcal{C}_{k}(\theta):=\int_{0}^{\theta} \cos ^{k} \psi d \psi-c_{k} \theta$ is a $2 \pi$-periodic function and it writes as $p_{k-1}(\cos \theta) \sin \theta$ with $p_{k-1}$ an even (odd) polynomial of degree $k-1$ when $k$ odd (even).

(e) $s_{k, \ell}:=\frac{1}{2 \pi} \int_{0}^{2 \pi} \sin ^{k} \psi \mathcal{S}_{\ell}(\psi) d \psi=\frac{(k-1) ! !}{k ! !} \frac{(\ell-1) ! !}{\ell ! !} \hat{\delta}_{k} \delta_{\ell}$ where $\delta_{\ell}= \begin{cases}0, & \text { if } \ell \text { is even, } \\ 1, & \text { if } \ell \text { is odd. }\end{cases}$

(f) $c_{k, \ell}:=\frac{1}{2 \pi} \int_{0}^{2 \pi} \cos ^{k} \psi \mathcal{C}_{\ell}(\psi) d \psi=0$.

(g) $\mathcal{S}_{k, \ell}(\theta):=\int_{0}^{\theta} \sin ^{k} \psi \mathcal{S}_{\ell}(\psi) d \psi-s_{k, \ell} \theta$ is a $2 \pi$-periodic function.

(h) $\mathcal{C}_{k, \ell}(\theta):=\int_{0}^{\theta} \cos ^{k} \psi \mathcal{C}_{\ell}(\psi) d \psi$ is a $2 \pi$-periodic function and it writes as $p_{k+\ell}(\cos \theta)$ with $p_{k+\ell}$ a polynomial of degree $k+\ell$.

(i) $s_{k, \ell}^{c}:=\frac{1}{2 \pi} \int_{0}^{2 \pi} \sin ^{k} \theta \cos \theta \mathcal{S}_{\ell}(\theta) d \theta=\frac{1}{k+1}\left(s_{k+1} s_{\ell}-s_{k+\ell+1}\right)$. 
(j) $\int_{0}^{\theta} \sin ^{k} \psi \cos \psi \mathcal{S}_{\ell}(\psi) d \psi-s_{k, \ell} \theta=\frac{1}{k+1}\left(\mathcal{S}_{\ell}(\theta) \sin ^{k+1} \theta-\right.$ $\left.\mathcal{S}_{k+\ell+1}(\theta)+s_{\ell} \mathcal{S}_{k+1}(\theta)\right)$ is a $2 \pi$-periodic function.

Proof. The properties (a), (b), (e), and (f) follow directly using iteratively the integration by parts method. Alternatively, the first two can be done also integrating by quarters, see for example [27]. The periodicity properties (c), (d), (g), and (h) follow easily because the correction by a multiple of $\theta$ converts the primitive of a periodic function into a periodic function. The second part of (d) follows also using iteratively the integration by parts method. Properties (i)-(j) follow by the integration by parts method and properties (a)-(b).

Lemma 2.7. The next properties hold.

(a) $\int_{0}^{2 \pi} \sin ^{k} \theta \mathcal{S}_{k}(\theta) d \theta=0$.

(b) $\int_{0}^{ \pm \pi} \mathcal{S}_{k}(\psi) d \psi= \pm \pi \frac{(k-1) ! !}{k ! !} \delta_{k}$.

(c) $\hat{s}_{k}:=\mathcal{S}_{k}(\pi)=\mathcal{S}_{k}(-\pi)=\frac{2(k-1) ! !}{k ! !} \delta_{k}$.

(d) $\hat{s}_{k, k}:=\mathcal{S}_{k, k}(\pi)=\mathcal{S}_{k, k}(-\pi)=\frac{1}{2}\left(\mathcal{S}_{k}(\pi)\right)^{2}=2\left(\frac{(k-1) ! !}{k ! !}\right)^{2} \delta_{k}$.

(e) $\gamma_{k}^{ \pm}:=\int_{0}^{ \pm \pi} \sin ^{k} \theta d \theta=\hat{s}_{k} \pm \pi s_{k}=( \pm 1)^{k-1} \frac{(k-1) ! !}{k ! !} \sigma_{k}$, where $\sigma_{k}= \begin{cases}\pi, & \text { if } k \text { is even } \\ 2, & \text { if } k \text { is odd. }\end{cases}$

(f) $\mathcal{C}_{k, \ell}(\pi)=c_{k} \mathcal{C}_{0, \ell}(\pi)+c_{\ell} \mathcal{C}_{0, k}(\pi)-\mathcal{C}_{\ell, k}(\pi)$, for $k+\ell$ odd.

(g) $\mathcal{C}_{k, \ell}(\pi)=\mathcal{C}_{k, \ell}(-\pi)$ and $\mathcal{C}_{k, \ell}(\pi)=\frac{1-(-1)^{k+\ell}}{(k+\ell) \ell}+\frac{\ell-1}{\ell} \mathcal{C}_{k, \ell-2}(\pi)$. Moreover, $\mathcal{C}_{k, \ell}(\pi)=0$ for $k+\ell$ even and $\mathcal{C}_{k, \ell}(\pi) \stackrel{\ell}{\neq} 0$ for $k+\ell$ odd.

Proof. Property (a) follows directly using Lemma 2.6(e) because $\delta_{k} \hat{\delta}_{k}=0$ for every $k$. The other properties follow, as Lemma 2.6, using iteratively, if necessary, the integration by parts rule.

\section{Polynomial perturbation}

This section is devoted to prove Theorem 1.1. The proof follows directly from the next three results. In the first we get the existence of the upper bounds for perturbations of every order $N$. The second and the third give why they are reached for $N=1$ and $N=2$, respectively.

Proposition 3.1. The maximum number of zeros of the Melnikov function $M_{N}$ associated to family (2) is $n$ and $N n-1$ for $N=1$ and $N \geq 2$, respectively.

Proposition 3.2. Consider the system

$$
\begin{array}{cc}
X^{+}:\left(x^{\prime}, y^{\prime}\right)=\left(-y+\varepsilon \sum_{j=0}^{\left[\frac{n-1}{2}\right]} a_{2 j+1} x^{2 j+1},\right. & \\
\left.x+\varepsilon \sum_{j=0}^{\left[\frac{n}{2}\right]} a_{2 j} x^{2 j}\right), & \text { if } y>0, \\
X^{-}:\left(x^{\prime}, y^{\prime}\right)=(-y, x), & \text { if } y<0 .
\end{array}
$$

The function $M_{1}(\rho)$ is a complete polynomial of degree $n$ with arbitrary coefficients. In particular, there exist values of $a_{k}, k=1, \ldots, n$, such that it has $n$ simple zeros.

Proposition 3.3. Consider the system

$$
\begin{array}{ll}
X^{+}:\left(x^{\prime}, y^{\prime}\right)=\left(-y-\varepsilon x^{n}+\varepsilon^{2} \sum_{j=0}^{n} a_{j} x^{j}, x+\varepsilon \sum_{j=2}^{n} b_{j} x^{j}\right), & \text { if } x>0, \\
X^{-}:\left(x^{\prime}, y^{\prime}\right)=\left(-y-(-1)^{n} \varepsilon x^{n}, x\right), & \text { if } x<0 .
\end{array}
$$

The function $M_{1}(\rho)$ vanishes identically and the function $M_{2}(\rho)$ is a complete polynomial of degree $2 n-1$. In particular, there exist coefficients $a_{j}, j=0, \ldots, n$ and $b_{j}, j=2, \ldots, n$, such that the function $M_{2}(\rho)$ has exactly $2 n-1$ simple zeros.

Proof of Proposition 3.1. Following the procedure described in Section 2, we will prove first how are the expressions, as using polynomials in $r$, of the functions $F_{i}, S_{i}$ and $h_{i}$, and second, how are the half return maps corresponding to the vector fields of both sides of the separation line.

In polar coordinates, $x=r \cos \theta$ and $y=r \sin \theta$, system (2) becomes

$$
\left\{\begin{aligned}
r r^{\prime}= & \sum_{i=1}^{N} \varepsilon^{i}\left(r \cos \theta f_{i}^{ \pm}(r \cos \theta, r \sin \theta)\right. \\
& \left.+r \sin \theta g_{i}^{ \pm}(r \cos \theta, r \sin \theta)\right), \\
r^{2} \theta^{\prime}= & 1+\sum_{i=1}^{N} \varepsilon^{i}\left(r \cos \theta g_{i}^{ \pm}(r \cos \theta, r \sin \theta)\right. \\
& \left.-r \sin \theta f_{i}^{ \pm}(r \cos \theta, r \sin \theta)\right) .
\end{aligned}\right.
$$

This system of equations writes, using differential one-forms, as

$\omega^{ \pm}=r \theta^{\prime} d r-r r^{\prime} d \theta=r d r+\sum_{i=1}^{N} \varepsilon^{i} \omega_{i}^{ \pm}=0$.

To simplify the notation, we use $p_{n}(r)$ to mean a polynomial of degree $n$ in the variable $r$. We do not take into account the dependence in the other variables or parameters. Then, with this notation, we can write $f_{i}^{ \pm}(r \cos \theta, r \sin \theta)=p_{n}(r), g_{i}^{ \pm}(r \cos \theta$, $r \sin \theta)=p_{n}(r)$, and $\omega_{i}^{ \pm}=p_{n}(r) d r+r p_{n}(r) d \theta$.

From Lemma 2.2, and considering $-\omega_{1}^{ \pm}=\alpha_{1}^{ \pm}(r, \theta) d r+$ $\beta_{1}^{ \pm}(r, \theta) d \theta$, we have

$$
\begin{aligned}
F_{1}^{ \pm}(r) & =\frac{1}{2 \pi} \int_{0}^{2 \pi} \beta_{1}^{ \pm}(r, \psi) d \psi=r p_{n}(r), \\
S_{1}^{ \pm}(r, \theta) & =\int_{0}^{\theta} \beta_{1}^{ \pm}(r, \psi) d \psi-F_{1}^{ \pm}(r) \theta=r p_{n}(r), \\
h_{1}^{ \pm}(r, \theta) & =\frac{1}{r}\left(\alpha_{1}^{ \pm}(r, \theta)-\frac{\partial S_{1}^{ \pm}}{\partial r}(r, \theta)\right)=p_{n-1}(r) .
\end{aligned}
$$

Here we observe that, according to Remark 2.4, the functions $F_{k}^{ \pm}$, $S_{k}^{ \pm}$, and $h_{k}^{ \pm}$given in Lemma 2.2 are not unique and can be chosen in such a way that they are polynomials. Now we prove by induction in $N$ that the functions $F_{k}^{ \pm}, S_{k}^{ \pm}$and $h_{k}^{ \pm}$associated to Eq. (11), of order $N$, can be written as

$$
\begin{aligned}
F_{k}^{ \pm}(r) & =r p_{k(n-1)+1}(r), \\
S_{k}^{ \pm}(r, \theta) & =r p_{k(n-1)+1}(r), \\
h_{k}^{ \pm}(r, \theta) & =p_{k(n-1)}(r),
\end{aligned}
$$

for $k=1, \ldots, N$. If we consider a perturbation of order $N+1$ in (11), then the functions $F_{k}^{ \pm}, S_{k}^{ \pm}$, and $h_{k}^{ \pm}$for $k=1, \ldots, N$ are, clearly, the same functions as the ones obtained for a perturbation of order $N$ in (11). Consequently, we only need to show how they are for $k=N+1$. In this case,

$$
\begin{aligned}
-\Omega_{N+1}^{ \pm}= & \alpha_{N+1}^{ \pm} d r+\beta_{N+1}^{ \pm} d \theta=-\left(\omega_{N+1}^{ \pm}+h_{1}^{ \pm} \omega_{N}+\cdots+h_{N}^{ \pm} \omega_{1}\right) \\
= & \left(p_{n}(r) d r+r p_{n}(r) d \theta\right)\left(1+p_{n-1}(r)+p_{2(n-1)}(r)\right. \\
& \left.+\cdots+p_{N(n-1)}(r)\right) \\
= & p_{N(n-1)+n}(r) d r+r p_{N(n-1)+n}(r) d \theta \\
= & p_{(N+1)(n-1)+1}(r) d r+r p_{(N+1)(n-1)+1}(r) d \theta
\end{aligned}
$$


So,

$$
\begin{aligned}
F_{N+1}^{ \pm}(r) & =\frac{1}{2 \pi} \int_{0}^{2 \pi} \beta_{N+1}^{ \pm}(r, \psi) d \psi=r p_{(N+1)(n-1)+1}(r), \\
S_{N+1}^{ \pm}(r, \theta) & =\int_{0}^{\theta} \beta_{N+1}^{ \pm}(r, \psi) d \psi-F_{N+1}^{ \pm}(r) \theta=r p_{(N+1)(n-1)+1}(r), \\
h_{N+1}^{ \pm}(r, \theta) & =\frac{1}{r}\left(\alpha_{N+1}^{ \pm}(r, \theta)-\frac{\partial S_{N+1}^{ \pm}}{\partial r}(r, \theta)\right)=p_{(N+1)(n-1)}(r) .
\end{aligned}
$$

Now we can continue proving which are the expressions of the coefficients in $\varepsilon$ of the half return map, that is, $r_{k}^{ \pm}$, for $k=1, \ldots, N$.

For $N=1$, the proof follows using Proposition 2.3, as we have

$r_{1}^{ \pm}(\theta, \rho)=\frac{1}{\rho}\left(S_{1}^{ \pm}(\rho, \theta)-F_{1}^{ \pm}(\rho) \theta\right)=\frac{1}{\rho} \rho p_{n}(\rho)=p_{n}(\rho)$.

For $N=2$ we write

$$
\begin{aligned}
\rho r_{2}^{ \pm}(\theta, \rho)= & -\frac{1}{2}\left(r_{1}^{ \pm}(\theta, \rho)\right)^{2}+S_{2}^{ \pm}(\rho, \theta)+D_{1} S_{1}^{ \pm}(\rho, \theta) r_{1}^{ \pm}(\theta, \rho) \\
& +F_{2}^{ \pm}(\rho) \theta+\left(F_{1}^{ \pm}\right)^{\prime}(\rho) \int_{0}^{\theta} r_{1}^{ \pm}(\psi, \rho) d \psi,
\end{aligned}
$$

with $D_{1} S_{1}^{ \pm}(\rho, \theta)=\left.\frac{\partial}{\partial r} S_{1}^{ \pm}(r, \theta)\right|_{r=\rho}$. It can be seen easily, from (12), that $r_{2}^{ \pm}(\theta, \rho)=p_{2 n-1}(\rho)$.

Next we will prove by induction in $N$ that

$r_{N}^{ \pm}(\theta, \rho)=\frac{p_{N n-1}(\rho)}{\rho^{N-2}}$

We assume that (13) holds for $k=2, \ldots, N$. According to Proposition 2.3 we have that $\rho r_{N+1}^{ \pm}(\theta, \rho)$ is given as a sum of three terms.

The first one is

$$
\begin{aligned}
-\frac{1}{2} \sum_{i=1}^{N} r_{i}^{ \pm}(\theta, \rho) r_{N+1-i}^{ \pm}(\theta, \rho) & =\sum_{i=1}^{N} \frac{p_{i n-1}(\rho)}{\rho^{i-2}} \frac{p_{(N+1-i) n-1}(\rho)}{\rho^{(N+1-i)-2}} \\
& =\frac{p_{(N+1) n-2}(\rho)}{\rho^{N-3}}=\frac{p_{(N+1) n-1}(\rho)}{\rho^{N-2}} .
\end{aligned}
$$

The second term, using the notation $D_{1}^{j} S_{i}^{ \pm}(\rho, \theta)=\left.\frac{\partial^{j} S_{i}^{ \pm}}{\partial r^{j}}(r, \theta)\right|_{r=\rho}$, is given by

$$
\begin{aligned}
& S_{N+1}^{ \pm}(\rho, \theta)+\sum_{i=1}^{N} \sum_{j=1}^{N+1-i} D_{1}^{j} S_{i}^{ \pm}(\rho, \theta) \\
& \quad \times \sum_{\substack{m_{1}+2 m_{2}+\cdots+\ell m_{\ell}=N+1-i \\
m_{1}+m_{2}+\cdots+m_{\ell}=j}}\left(r_{1}^{ \pm}(\theta, \rho)\right)^{m_{1}}\left(r_{2}^{ \pm}(\theta, \rho)\right)^{m_{2}} \cdots\left(r_{\ell}^{ \pm}(\theta, \rho)\right)^{m_{\ell}} \\
&= \rho p_{(N+1)(n-1)+1}(\rho) \\
&+\frac{p_{i(n-1)+2-j}(\rho) \times p_{m_{1} n+m_{2}(2 n-1)+\cdots+m_{\ell}(\ell n-1)}(\rho)}{r^{m_{3}+2 m_{4}+3 m_{5}+\cdots+(\ell-2) m_{\ell}}} \\
&= \frac{\rho \rho^{N-2} p_{(N+1)(n-1)+1}(\rho)}{r^{N-2}} \\
&+\frac{p_{i(n-1)+2-j+n\left(m_{1}+2 m_{2}+\cdots+\ell m_{\ell}\right)-\left(m_{2}+\cdots+m_{\ell}\right)}(\rho)}{r^{\left(3 m_{3}+4 m_{4}+\cdots+\ell m_{\ell}\right)-2\left(m_{3}+m_{4}+\cdots+m_{\ell}\right)}} \\
&= \frac{p_{(N+1) n-1}(\rho)}{\rho^{N-2}}+\frac{p_{i(n-1)+2-j+n(N+1-i)-\left(j-m_{1}\right)}(\rho)}{\rho^{\left(N+1-i-m_{1}-2 m_{2}\right)-2\left(j-m_{1}-m_{2}\right)}} \\
&= \frac{p_{(N+1) n-1}(\rho)}{\rho^{N-2}}+\frac{p_{(N+1) n+2+m_{1}-i-2 j}(\rho)}{\rho^{N+1+m_{1}-i-2 j}} \\
&= \frac{p_{(N+1) n-1}(\rho)}{\rho^{N-2}}+\frac{\rho^{i+2 j-m_{1}-3}}{\rho^{i+2 j-m_{1}-3}} \frac{p_{(N+1) n+2+m_{1}-i-2 j}(\rho)}{\rho^{N+1+m_{1}-i-2 j}} \\
& p_{(N+1) n-1}(\rho) \\
& \rho^{N-2}
\end{aligned}
$$

The third term has the same expression as above changing $S$ by $F$ and integrating in the variable $\theta$, but there are no changes in the degrees as a function of $r$. So, as the three terms write in the same form, after division by $\rho$, we have

$r_{N+1}^{ \pm}(\theta, \rho)=\frac{p_{(N+1) n-1}(\rho)}{\rho^{(N+1)-2}}$.

Finally, from (13), the difference $r_{N}^{+}(\pi, \rho)-r_{N}^{-}(-\pi, \rho)$ also writes as $p_{N n-1}(\rho) / \rho^{N-2}$. Then, from Proposition 2.5 , the maximum number of positive zeros of $M_{N}(\rho)$ is $N n-1$.

Proof of Proposition 3.2. In polar coordinates, $x=r \cos \theta, y=$ $r \sin \theta$, the half return map of the vector field $X^{-}$is the identity. In particular $r_{1}^{-}(-\pi, \rho)=0$ and $M_{1}(\rho)=r_{1}^{+}(\pi, \rho)$. For simplify the reading we will omit in this proof the dependence of ${ }^{+}$because we only need to compute the half return map for $\theta \in(0, \pi)$. Then, in polar coordinates, the vector field $X^{+}$of $(10)$ writes

$$
\left\{\begin{aligned}
r^{\prime}= & \varepsilon\left(\sum_{j=0}^{n_{o}} a_{2 j+1} r^{2 j+1} \cos ^{2 j+2} \theta+\sum_{j=0}^{n_{e}} a_{2 j} r^{2 j} \cos ^{2 j} \theta \sin \theta\right), \\
\theta^{\prime}= & 1+\varepsilon\left(-\sum_{j=0}^{n_{o}} a_{2 j+1} r^{2 j} \cos ^{2 j+1} \theta \sin \theta\right. \\
& \left.+\sum_{j=0}^{n_{e}} a_{2 j} r^{2 j-1} \cos ^{2 j+1} \theta\right),
\end{aligned}\right.
$$

where $n_{o}=[(n-1) / 2]$ and $n_{e}=[n / 2]$. In terms of differential one-forms we write

$\omega=r \theta^{\prime} d r-r r^{\prime} d \theta=r d r+\varepsilon \omega_{1}$,

where

$-\omega_{1}=\alpha_{1}(r, \theta) d r+\beta_{1}(r, \theta) d \theta$,

with

$$
\begin{aligned}
& \alpha_{1}(r, \theta)=\sum_{j=0}^{n_{o}} a_{2 j+1} r^{2 j+1} \cos ^{2 j+1} \theta \sin \theta-\sum_{j=0}^{n_{e}} a_{2 j} r^{2 j} \cos ^{2 j+1} \theta, \\
& \beta_{1}(r, \theta)=\sum_{j=0}^{n_{o}} a_{2 j+1} r^{2 j+2} \cos ^{2 j+2} \theta+\sum_{j=0}^{n_{e}} a_{2 j} r^{2 j+1} \cos ^{2 j} \theta \sin \theta .
\end{aligned}
$$

According to Lemmas 2.2 and 2.6, and using $\int_{0}^{2 \pi} \cos ^{2 j+1} \psi$ $\sin \psi d \psi=0$, we have that

$$
\begin{aligned}
F_{1}(r)= & \frac{1}{2 \pi} \int_{0}^{2 \pi} \beta_{1}(r, \psi) d \psi=\sum_{j=0}^{n_{o}} a_{2 j+1} c_{2 j+2} r^{2 j+2}, \\
S_{1}(r, \theta)= & \int_{0}^{\theta} \beta_{1}(r, \psi) d \psi-F_{1}(r) \theta=\sum_{j=0}^{n_{o}} a_{2 j+1} r^{2 j+2} \mathcal{C}_{2 j+2}(\theta) \\
& -\sum_{j=0}^{n_{e}} \frac{a_{2 j}}{2 j+1} r^{2 j+1}\left(\cos ^{2 j+1} \theta-1\right) .
\end{aligned}
$$

Clearly $S_{1}$ is $2 \pi$-periodic in $\theta$, because the functions $\mathcal{C}_{2 j+2}(\theta)$ also are. From Proposition 2.3 we have that $r_{1}^{+}(\theta, \rho)=\left(S_{1}(\rho, \theta)+\right.$ $\left.F_{1}(\rho) \theta\right) / \rho$ and, from Lemma 2.7,

$r_{1}^{+}(\pi, \rho)=\sum_{j=0}^{n_{e}} \frac{2 a_{2 j}}{2 j+1} \rho^{2 j}+\sum_{j=0}^{n_{o}} a_{2 j+1} c_{2 j+2} \rho^{2 j+1}$,

with $c_{2 j+2} \neq 0$. Consequently, there exist perturbation values such that $M_{1}(\rho)=r_{1}^{+}(\pi, \rho)$ has $n$ simple zeros. Because it is a polynomial of degree $n=\max \left\{2 n_{o}+1,2 n_{e}\right\}$ with arbitrary coefficients. 
Proof of Proposition 3.3. First of all we consider the unified system

$X^{ \pm}:\left(x^{\prime}, y^{\prime}\right)=\left(-y+\delta^{ \pm} \varepsilon x^{n}+\varepsilon^{2} \sum_{j=0}^{n} a_{j}^{ \pm} x^{j}, x+\varepsilon \sum_{j=2}^{n} b_{j}^{ \pm} x^{j},\right)$

where $\delta^{+}=-1, a_{j}^{+}=a_{j}, b_{j}^{+}=b_{j}, \delta^{-}=-(-1)^{n}, a_{j}^{-}=0$, and $b_{j}^{-}=0$.

In polar coordinates, $x=r \cos \varphi, y=r \sin \varphi$, we get

$$
\left\{\begin{aligned}
r^{\prime}= & \varepsilon\left(\delta^{ \pm} r^{n} \cos ^{n+1} \varphi+\sum_{j=2}^{n} b_{j}^{ \pm} r^{j} \cos ^{j} \varphi \sin \varphi\right) \\
& +\varepsilon^{2} \sum_{j=0}^{n} a_{j}^{ \pm} r^{j} \cos ^{j+1} \varphi, \\
\varphi^{\prime}= & 1-\varepsilon\left(\delta^{ \pm} r^{n-1} \cos ^{n} \varphi \sin \varphi-\sum_{j=2}^{n} b_{j}^{ \pm} r^{j-1} \cos ^{j+1} \varphi\right) \\
& -\varepsilon^{2} \sum_{j=0}^{n} a_{j}^{ \pm} r^{j-1} \cos ^{j} \varphi \sin \varphi .
\end{aligned}\right.
$$

First we make a rotation of angle $\pi / 2$ in order to have the discontinuity line as the horizontal axis. With the change $\theta=\varphi+\pi / 2$, $\cos \varphi=\sin \theta$ and $\sin \varphi=-\cos \theta$, system (14) becomes

$$
\left\{\begin{aligned}
r^{\prime}= & \varepsilon\left(\delta^{ \pm} r^{n} \sin ^{n+1} \theta-\sum_{j=2}^{n} b_{j}^{ \pm} r^{j} \sin ^{j} \theta \cos \theta\right) \\
& +\varepsilon^{2} \sum_{j=0}^{n} a_{j}^{ \pm} r^{j} \sin ^{j+1} \theta, \\
\theta^{\prime}= & 1+\varepsilon\left(\delta^{ \pm} r^{n-1} \sin ^{n} \theta \cos \theta+\sum_{j=2}^{n} b_{j}^{ \pm} r^{j-1} \sin ^{j+1} \theta\right) \\
& +\varepsilon^{2} \sum_{j=0}^{n} a_{j}^{ \pm} r^{j-1} \sin ^{j} \theta \cos \theta .
\end{aligned}\right.
$$

or, using differential one-forms, we have

$\omega^{ \pm}=r \theta^{\prime} d r-r r^{\prime} d \theta=r d r+\varepsilon \omega_{1}^{ \pm}+\varepsilon^{2} \omega_{2}^{ \pm}=0$,

where

$-\omega_{1}^{ \pm}=\alpha_{1}^{ \pm}(r, \theta) d r+\beta_{1}^{ \pm}(r, \theta) d \theta$

with

$\alpha_{1}^{ \pm}(r, \theta)=-\delta^{ \pm} r^{n} \sin ^{n} \theta \cos \theta-\sum_{j=2}^{n} b_{j}^{ \pm} r^{j} \sin ^{j+1} \theta$,

$\beta_{1}^{ \pm}(r, \theta)=\delta^{ \pm} r^{n+1} \sin ^{n+1} \theta-\sum_{j=2}^{n} b_{j}^{ \pm} r^{j+1} \sin ^{j} \theta \cos \theta$

The explicit expression of $\omega_{2}^{ \pm}$will appear later when we study the perturbation using the second order terms.

For the first order study, according to Lemmas 2.2 and 2.6, we have

$$
\begin{aligned}
F_{1}^{ \pm}(r)= & \frac{1}{2 \pi} \int_{0}^{2 \pi} \beta_{1}^{ \pm}(r, \psi) d \psi=\frac{\delta^{ \pm} r^{n+1}}{2 \pi} \int_{0}^{2 \pi} \sin ^{n+1} \psi d \psi \\
= & \delta^{ \pm} r^{n+1} s_{n+1}, \\
S_{1}^{ \pm}(r, \theta)= & \int_{0}^{\theta} \beta_{1}^{ \pm}(r, \psi) d \psi-F_{1}^{ \pm}(r) \theta=\delta^{ \pm} r^{n+1} \mathcal{S}_{n+1}(\theta) \\
& -\sum_{j=2}^{n} b_{j}^{ \pm} \frac{1}{j+1} r^{j+1} \sin ^{j+1} \theta .
\end{aligned}
$$

Clearly $S_{1}^{ \pm}$is $2 \pi$-periodic because $\mathcal{S}_{n+1}(\theta)$ also is. From Proposition 2.3 we have that $r_{1}^{ \pm}(\theta, \rho)=\left(S_{1}^{ \pm}(\rho, \theta)+F_{1}^{ \pm}(\rho) \theta\right) / \rho$. It is clear, from Lemma 2.7, that $S_{1}^{+}(\rho, \pi)-S_{1}^{-}(\rho,-\pi)=\left(\delta^{+}-\delta^{-}\right) \hat{s}_{n+1} \rho^{n+1}$ and $F_{1}^{+}(\rho) \pi-F_{1}^{-}(\rho)(-\pi)=\pi \rho^{n+1}\left(\delta^{+}+\delta^{-}\right) s_{n+1}$. We observe that, when $n$ is even, $\delta^{+}=\delta^{-}$and $s_{n+1}=0$, and when $n$ is odd, $\delta^{+}=-\delta^{-}$and $\hat{s}_{n+1}=0$. So, the first Melnikov function is

$M_{1}(\rho)=r_{1}^{+}(\pi, \rho)-r_{1}^{-}(-\pi, \rho)=0$.

The next step is the computation of the second Melnikov function $M_{2}(\rho)$. In order to get it, first we compute the function $h_{1}^{ \pm}(r, \theta)$ using Lemma 2.2,

$$
\begin{aligned}
h_{1}^{ \pm}(r, \theta) & =\frac{1}{r}\left(\alpha_{1}^{ \pm}(r, \theta)-\frac{\partial S_{1}^{ \pm}}{\partial r}(r, \theta)\right) \\
& =-\delta^{ \pm} r^{n-1}\left(\sin ^{n} \theta \cos \theta+(n+1) \mathcal{S}_{n+1}(\theta)\right) .
\end{aligned}
$$

The study of the second order terms, also using the decomposition of Lemma 2.2, uses

$$
-\omega_{1}^{ \pm} h_{1}^{ \pm}-\omega_{2}^{ \pm}=\alpha_{2}^{ \pm}(r, \theta) d r+\beta_{2}^{ \pm}(r, \theta) d \theta
$$

with

$$
\begin{aligned}
\beta_{2}^{ \pm}(r, \theta) & =\sum_{j=0}^{n} a_{j}^{ \pm} r^{j+1} \sin ^{j+1} \theta+\left(\delta^{ \pm} r^{n+1} \sin ^{n+1} \theta\right. \\
& \left.-\sum_{j=2}^{n} b_{j}^{ \pm} r^{j+1} \sin ^{j} \theta \cos \theta\right) \\
& \times\left(-\delta^{ \pm} r^{n-1}\left(\sin ^{n} \theta \cos \theta+(n+1) \mathcal{S}_{n+1}(\theta)\right)\right) \\
& =\sum_{j=0}^{n} a_{j}^{ \pm} r^{j+1} \sin ^{j+1} \theta-\left(\delta^{ \pm}\right)^{2} r^{2 n}\left(\sin ^{2 n+1} \theta \cos \theta\right. \\
& \left.+(n+1) \sin ^{n+1} \theta \mathcal{S}_{n+1}(\theta)\right) \\
& +\sum_{j=2}^{n} \delta^{ \pm} b_{j}^{ \pm} r^{n+j} \sin ^{n+j} \theta \cos ^{2} \theta+(n+1) \\
& \times \sum_{j=2}^{n} \delta^{ \pm} b_{j}^{ \pm} r^{n+j} \sin ^{j} \theta \cos \theta \mathcal{S}_{n+1}(\theta) .
\end{aligned}
$$

We have not written the expression of $\alpha^{ \pm}$because they are not needed for the computation of $r_{2}^{ \pm}(\theta, \rho)$.

Using again Lemma 2.2 and the properties of Lemma 2.6, and $\int_{0}^{2 \pi} \sin ^{2 n+1} \theta \cos \theta d \theta=0$ we have that

$$
\begin{aligned}
F_{2}^{ \pm}(r)= & \frac{1}{2 \pi} \int_{0}^{2 \pi} \beta_{2}^{ \pm}(r, \psi) d \psi=\sum_{j=0}^{n} a_{j}^{ \pm} r^{j+1} s_{j+1} \\
& +\sum_{j=2}^{n} \delta^{ \pm} b_{j}^{ \pm} r^{n+j} s_{n+j} \\
& -\sum_{j=2}^{n} \delta^{ \pm} b_{j}^{ \pm} r^{n+j} s_{n+j+2}+(n+1) \sum_{j=2}^{n} \delta^{ \pm} b_{j}^{ \pm} r^{n+j} \\
& \times \frac{1}{j+1}\left(s_{n+1} s_{j+1}-s_{n+j+2}\right) .
\end{aligned}
$$

So,

$$
\begin{aligned}
F_{2}^{ \pm}(r)= & \sum_{j=0}^{n} a_{j}^{ \pm} r^{j+1} s_{j+1}+\sum_{j=2}^{n} \delta^{ \pm} b_{j}^{ \pm} r^{n+j} \\
& \times\left(s_{n+j}-s_{n+j+2}+\frac{n+1}{j+1}\left(s_{n+1} s_{j+1}-s_{n+j+2}\right)\right) .
\end{aligned}
$$


Again from Lemmas 2.2 and 2.6, we have

$$
\begin{aligned}
S_{2}^{ \pm}(r, \theta) & =\int_{0}^{\theta} \beta_{2}^{ \pm}(r, \psi) d \psi-F_{2}^{ \pm}(r) \theta \\
& =\sum_{j=0}^{n} a_{j}^{ \pm} r^{j+1} \mathcal{S}_{j+1}(\theta)-\left(\delta^{ \pm}\right)^{2} r^{2 n} \\
& \times\left(\frac{1}{2 n+2} \sin ^{2 n+2} \theta+(n+1) \mathcal{S}_{n+1, n+1}(\theta)\right) \\
& +\sum_{j=2}^{n} \delta^{ \pm} b_{j}^{ \pm} r^{n+j}\left(\mathcal{S}_{n+j}(\theta)-\mathcal{S}_{n+j+2}(\theta)\right) \\
& +(n+1) \sum_{j=2}^{n} \delta^{ \pm} b_{j}^{ \pm} r^{n+j} \frac{1}{j+1} \\
& \times\left(\mathcal{S}_{n+1}(\theta) \sin ^{j+1} \theta-\mathcal{S}_{n+j+2}(\theta)+s_{n+1} \mathcal{S}_{j+1}(\theta)\right) .
\end{aligned}
$$

Finally, from Propositions 2.3 and 2.5, the second Melnikov function is $M_{2}(\rho)=r_{2}^{+}(\pi, \rho)-r_{2}^{-}(-\pi, \rho)$. We compute this difference term by term. The first and third terms vanish because $r_{1}^{+}(\pi, \rho)-r_{1}^{-}(-\pi, \rho)=0$ and, from (15) and (16),

$\left.\frac{\partial S_{1}^{+}(r, \pi)}{\partial r}\right|_{r=\rho}-\left.\frac{\partial S_{1}^{-}(r,-\pi)}{\partial r}\right|_{r=\rho}=(n+1)\left(\delta^{+}-\delta^{-}\right) \hat{s}_{n+1}=0$.

The above expression vanishes using the same argument explained in the paragraph before (16). From Lemma 2.7 we have

$$
\begin{aligned}
\frac{1}{\rho}\left(F_{1}^{+}\right)^{\prime}(\rho) \int_{0}^{\pi} r_{1}^{+}(\psi, \rho) d \psi & =\left(\delta^{+}\right)^{2}(n+1) \rho^{2 n-1} s_{n+1} \\
& \times\left(\int_{0}^{\pi} \mathcal{S}_{n+1}(\psi) d \psi-s_{n+1} \frac{\pi^{2}}{2}\right) \\
& +\sum_{j=2}^{n} \frac{n+1}{j+1} b_{j} \rho^{n+j-1} s_{n+1} \\
& \times\left(\hat{s}_{j+1}+\pi s_{j+1}\right) \\
& =-\frac{(n+1) \pi^{2}}{2} s_{n+1}^{2} \rho^{2 n-1} \\
& +\sum_{j=2}^{n} \frac{n+1}{j+1} b_{j} s_{n+1} \gamma_{j+1}^{+} \rho^{n+j-1} .
\end{aligned}
$$

In the last equality, the first term vanishes because, from the expressions of Lemma 2.6, $s_{n+1}$ is a multiple of $\delta_{n+1}$ and the integral is a multiple of $\hat{\delta}_{n+1}$. Now, doing similar simplifications, we get

$$
\begin{aligned}
\frac{1}{\rho}\left(F_{1}^{-}\right)^{\prime}(\rho) \int_{0}^{-\pi} r_{1}^{-}(\psi, \rho) d \psi= & \left(\delta^{-}\right)^{2}(n+1) \rho^{2 n-1} s_{n+1} \\
& \times\left(\int_{0}^{-\pi} \mathcal{S}_{n+1}(\psi) d \psi-s_{n+1} \frac{\pi^{2}}{2}\right) \\
= & -\frac{(n+1) \pi^{2}}{2} s_{n+1}^{2} \rho^{2 n-1} .
\end{aligned}
$$

The contribution of the last term, in the expressions of $r_{2}^{ \pm}$, to $M_{2}$ is obtained from the difference of (17) and (18). Hence, the first part of the second Melnikov function writes as

$$
\sum_{j=2}^{n} \frac{n+1}{j+1} b_{j} s_{n+1} \gamma_{j+1}^{+} \rho^{n+j-1} \text {. }
$$

Now, we continue computing the second and fourth terms. Adding the following two expressions

$$
\frac{1}{\rho} S_{2}^{+}(\rho, \pi)=\sum_{j=2}^{n} b_{j} \rho^{n+j-1}\left(\frac{n+j+2}{j+1} \hat{s}_{n+j+2}-\hat{s}_{n+j}\right.
$$

$$
\begin{aligned}
& \left.-\frac{n+1}{j+1} s_{n+1} \hat{s}_{j+1}\right) \\
& +\sum_{j=0}^{n} a_{j} \rho^{j} \hat{s}_{j+1}-(n+1) \rho^{2 n-1} \hat{s}_{n+1, n+1}, \\
\frac{\pi}{\rho} F_{2}^{+}(\rho)= & \sum_{j=2}^{n} \pi b_{j} \rho^{n+j-1}\left(\frac{n+j+2}{j+1} s_{n+j+2}-s_{n+j}\right. \\
& \left.-\frac{n+1}{j+1} s_{n+1} s_{j+1}\right) \\
& +\sum_{j=0}^{n} \pi a_{j} \rho^{j} s_{j+1},
\end{aligned}
$$

we obtain that the contribution of that terms, corresponding to the vector field $X^{+}$, is

$$
\begin{aligned}
& \sum_{j=0}^{n} a_{j} \rho^{j} \gamma_{j+1}+\sum_{j=2}^{n} b_{j} \rho^{n+j-1}\left(\frac{n+j+2}{j+1} \gamma_{n+j+2}^{+}\right. \\
& \left.-\gamma_{n+j}^{+}-\frac{n+1}{j+1} s_{n+1} \gamma_{j+1}^{+}\right) \\
& -(n+1) \rho^{2 n-1} \hat{s}_{n+1, n+1} .
\end{aligned}
$$

The contribution corresponding to the vector field $X^{-}$is the sum of the following two terms:

$$
\begin{aligned}
\frac{1}{\rho} S_{2}^{-}(\rho,-\pi) & =-(n+1) \rho^{2 n-1} \hat{S}_{n+1, n+1}, \\
-\frac{\pi}{\rho} F_{2}^{-}(\rho) & =0,
\end{aligned}
$$

that is, after adding them, only

$$
-(n+1) \rho^{2 n-1} \hat{s}_{n+1, n+1} \text {. }
$$

Consequently the difference of Eqs. (20) and (21) provides the second part of the second Melnikov function:

$$
\begin{aligned}
& \sum_{j=0}^{n} a_{j} \rho^{j} \gamma_{j+1}^{+}+\sum_{j=2}^{n} b_{j} \rho^{n+j-1}\left(\frac{n+j+2}{j+1} \gamma_{n+j+2}^{+}-\gamma_{n+j}^{+}\right. \\
& \left.\quad-\frac{n+1}{j+1} s_{n+1} \gamma_{j+1}^{+}\right) .
\end{aligned}
$$

Finally, adding (19) and (22) and using that $\sigma_{n+j+2}=\sigma_{n+j}$ and

$\frac{n+j+1}{j+1} \gamma_{n+j}^{+}-\gamma_{n+j}^{+}=\frac{n}{j+1} \gamma_{n+j}^{+}$,

we obtain the expression for the second Melnikov function

$M_{2}(\rho)=\sum_{j=0}^{n} a_{j} \gamma_{j+1}^{+} \rho^{j}+\sum_{j=2}^{n} \frac{n}{j+1} b_{j} \gamma_{n+j}^{+} \rho^{n+j-1}$,

with $\gamma_{k}^{+} \neq 0$, for every $k$, see also Lemma 2.7 .

The proof finishes because $M_{2}$ is a complete polynomial of degree $2 n-1$ with arbitrary coefficients. So, we can choose them such that $M_{2}(\rho)$ has exactly $2 n-1$ simple roots.

\section{Perturbations of order one and two for some Liénard families}

This section is devoted to prove Theorem 1.2. It is a direct consequence of the next two results.

Proposition 4.1. Consider the piecewise Liénard system

$\left(x^{\prime}, y^{\prime}\right)=\left(-y+\varepsilon f_{n}^{ \pm}(x), x\right)$, 
defined in $\Sigma_{0}^{ \pm}=\left\{(x, y) \in \mathbb{R}^{2} ; \pm y>0\right\}$, where $f_{n}^{ \pm}(x)=\sum_{j=0}^{n} a_{j}^{ \pm} x^{j}$. The first Melnikov function writes as $M_{1, n}(\rho)=\rho p_{m}\left(\rho^{2}\right)$ for a given polynomial $p_{m}$ of degree $m=[(n-1) / 2]$. Then, it has at most $m$ positive zeros. Moreover, there exist values of the parameters $a_{j}^{ \pm}$such that $M_{1, n}$ has exactly $m$ positive simple zeros. Additionally, $M_{1, n} \equiv 0$ if and only if $a_{2 k-1}^{+}=-a_{2 k-1}^{-}$for $k=1, \ldots, m+1$.

Proposition 4.2. Consider the piecewise Liénard system

$\left(x^{\prime}, y^{\prime}\right)=\left(-y+\varepsilon f_{n}^{ \pm}(x)+\varepsilon^{2} g_{n}^{ \pm}(x), x\right)$,

defined in $\Sigma_{0}^{ \pm}=\left\{(x, y) \in \mathbb{R}^{2} ; \pm y>0\right\}$, where $f_{n}^{ \pm}(x)=\sum_{j=0}^{n} a_{j}^{ \pm} x^{j}$ and $g_{n}^{ \pm}(x)=\sum_{j=0}^{n} b_{j}^{ \pm} x^{j}$, satisfying $a_{2 k-1}^{+}=-a_{2 k-1}^{-}$for $k=$ $1, \ldots, m+1$, where $m=[(n-1) / 2]$. Then, the first Melnikov function vanishes identically, $M_{1, n} \equiv 0$, and the second Melnikov function writes as $M_{2, n}(\rho)=\rho p_{m}\left(\rho^{2}\right)+q_{n-1}\left(\rho^{2}\right)$ for given polynomials $p_{m}$ and $q_{n-1}$ of degrees $m=[(n-1) / 2]$ and $n-1$, respectively. Then, it has at most $m+n$ positive zeros. Moreover, there exist values of the parameters $a_{j}^{ \pm}$and $b_{j}^{ \pm}$such that $M_{2, n}$ has exactly $m+n$ positive simple zeros.

Proof of Proposition 4.1. We start writing system (23), with polar coordinates, as the piecewise one-form

$$
\left\{\begin{array}{l}
d H+\varepsilon \omega_{1, n}^{+}=0 \text { if } \theta \in[0, \pi), \\
d H+\varepsilon \omega_{1, n}^{-}=0 \text { if } \theta \in[\pi, 2 \pi),
\end{array}\right.
$$

where $H=r^{2} / 2$ and $-\omega_{1, n}^{ \pm}=\alpha_{1, n}^{ \pm}(r, \theta) d r+\beta_{1, n}^{ \pm}(r, \theta) d \theta$, with

$\alpha_{1, n}^{ \pm}(r, \theta)=f_{n}^{ \pm}(r \cos \theta) \sin \theta=\sum_{j=0}^{n} a_{j}^{ \pm} r^{j} \cos ^{j} \theta \sin \theta$,

$\beta_{1, n}^{ \pm}(r, \theta)=f_{n}^{ \pm}(r \cos \theta) r \cos \theta=\sum_{j=0}^{n} a_{j}^{ \pm} r^{j+1} \cos ^{j+1} \theta$.

Using Lemma 2.2 and Proposition 2.3, from the above expressions, we get

$r_{1, n}^{ \pm}(\theta):=\rho r_{1, n}^{ \pm}(\theta)=F_{1, n}^{ \pm}(\rho) \theta+S_{1, n}^{ \pm}(\rho, \theta)$,

where

$F_{1, n}^{ \pm}(r)=\frac{1}{2 \pi} \int_{0}^{2 \pi} \beta_{1, n}^{ \pm}(r, \theta) d \theta$

and

$S_{1, n}^{ \pm}(r, \theta)=\int_{0}^{\theta} \beta_{1, n}^{ \pm}(r, \psi) d \psi-F_{1, n}^{ \pm}(r) \theta$.

Now, we prove that the functions $F_{1, n}^{ \pm}(r)$ and $S_{1, n}^{ \pm}(r, \theta)$ are polynomials in $r$ with constant coefficients and trigonometric functions, respectively.

For $n=0$, we have $\alpha_{1,0}^{ \pm}=a_{0}^{ \pm} \sin \theta$ and $\beta_{1,0}^{ \pm}=b_{0}^{ \pm} r \cos \theta$. Hence, using Proposition 2.5, $M_{1,0}(\rho)=0$, because $F_{1,0}^{ \pm}(r)=0$ and $S_{1,0}(r, \theta)=a_{0}^{ \pm} r \sin \theta$.

For any $n$, we can write

$$
\begin{aligned}
F_{1, n+1}^{ \pm}(r) & =\sum_{k=1}^{\left[\frac{n+1}{2}\right]} a_{2 k-1}^{ \pm} c_{2 k} r^{2 k}+a_{n+1}^{ \pm} c_{n+2} r^{n+2} \\
& =F_{1, n}^{ \pm}(r)+a_{n+1}^{ \pm} c_{n+2} r^{n+2},
\end{aligned}
$$

because, from Lemma 2.6, we have $c_{j}=0$ for every $j$ odd. We remark that the monomial in $r^{n+2}$ only appears when $n$ is even.
Additionally, we can write

$$
\begin{aligned}
S_{1, n+1}^{ \pm}(r, \theta)= & \sum_{j=0}^{n+1} a_{j}^{ \pm} r^{j+1} \int_{0}^{\theta} \cos ^{j+1} \psi d \psi-F_{1, n+1}(r) \theta \\
= & \left(\sum_{j=0}^{n} a_{j}^{ \pm} r^{j+1} \int_{0}^{\theta} \cos ^{j+1} \psi d \psi-F_{1, n}(r) \theta\right) \\
& +\left(a_{n+1}^{ \pm} r^{n+2} \int_{0}^{\theta} \cos ^{n+2} \psi d \psi-a_{n+1}^{ \pm} c_{n+2} r^{n+2} \theta\right) \\
= & S_{1, n}^{ \pm}(r, \theta)+a_{n+1}^{ \pm}\left(\int_{0}^{\theta} \cos ^{n+2} \psi d \psi-c_{n+2} \theta\right) r^{n+2}
\end{aligned}
$$

and, consequently,

$S_{1, n+1}^{ \pm}(r, \theta)=S_{1, n}^{ \pm}(r, \theta)+a_{n+1}^{ \pm} \mathcal{C}_{n+2}(\theta) r^{n+2}$.

The above expression can also be written as

$S_{1, n}^{ \pm}(r, \theta)=r \sin \theta G_{n}(r, \cos \theta)$,

where $G_{n}(r, \cos \theta)$ is a polynomial of degree $n$ in $r$ which coefficients are polynomials in $\cos \theta$. This property follows by induction on $n$ using also Lemma 2.6(d).

Straightforward computations show, from (26), (27), and (28), that

$\rho r_{1, n+1}^{ \pm}(\theta, \rho)=\rho r_{1, n}^{ \pm}(\theta, \rho)+a_{n+1}^{ \pm}\left(\mathcal{C}_{n+2}(\theta)+c_{n+2} \theta\right) \rho^{n+2}$,

with $r_{1,0}^{ \pm}(\theta)=a_{0}^{ \pm} \sin \theta$, and, consequently,

$r_{1, n}^{ \pm}(\theta, \rho)=\sum_{j=0}^{n} a_{j}^{ \pm}\left(\mathcal{C}_{j+1}(\theta)+c_{j+1} \theta\right) \rho^{j}$.

Therefore, using also Proposition 2.5, we can write the first Melnikov function as

$$
\begin{aligned}
M_{1, n+1}(\rho) & =r_{1, n+1}^{+}(\pi, \rho)-r_{1, n+1}^{-}(-\pi, \rho) \\
& =r_{1, n}^{+}(\pi, \rho)-r_{1, n}^{-}(-\pi, \rho)+\pi\left(a_{n+1}^{+}+a_{n+1}^{-}\right) c_{n+2} \rho^{n+1} \\
& =M_{1, n}(\rho)+\pi\left(a_{n+1}^{+}+a_{n+1}^{-}\right) c_{n+2} \rho^{n+1} .
\end{aligned}
$$

Then, using Lemma 2.6, the function $M_{1, n}$ is a polynomial in $\rho$ of degree $2 m+1$, with $m=[(n-1) / 2]$, because $M_{1,0}(\rho) \equiv 0$, $c_{j}=0$ for $j$ odd, and $c_{j} \neq 0$ for $j$ even. More concretely, it writes as $M_{1, n}(\rho)=\rho p_{m}\left(\rho^{2}\right)$ with

$$
\begin{aligned}
p_{m}(\rho)= & \pi\left(c_{2}\left(a_{1}^{+}+a_{1}^{-}\right)+c_{4}\left(a_{3}^{+}+a_{3}^{-}\right) \rho\right. \\
& \left.+\cdots+c_{2 m+2}\left(a_{2 m+1}^{+}+a_{2 m+1}^{-}\right) \rho^{m}\right),
\end{aligned}
$$

where $c_{2 k} \neq 0$ for $k=1, \ldots, m+1$.

Clearly, from the above expression, all the statements follow. The function $M_{1, n}$ has at most $m$ zeros and we can choose all the perturbation parameters in order to get these zeros as simple ones. Moreover, $M_{1, n} \equiv 0$ if and only if

$a_{2 k-1}^{+}=-a_{2 k-1}^{-} \quad$ for $\quad k=1, \ldots, m+1$.

Proof of Proposition 4.2. First, we provide a short description of the proof, with the main properties. Then, we proceed to detail all the steps carefully.

From Proposition 4.1 we know that the first Melnikov function vanishes identically when $a_{2 k-1}^{+}+a_{2 k-1}^{-}=0$. In the proof we will refer this property as $\left(M_{1}\right)$. The second Melnikov function writes as a sum of two functions,

$M_{2, n}(\rho)=M_{2, n}^{(1)}(\rho)+M_{2, n}^{(2)}(\rho)$,

that they correspond to the perturbation parameters of first and second order, $a_{j}$ and $b_{j}$, respectively in (24). We can also use 
Proposition 4.1 , changing $\varepsilon$ to $\varepsilon^{2}$, to see that $M_{2, n}^{(2)}(\rho)=\rho p_{m}\left(\rho^{2}\right)$ for some polynomial $p_{m}$, of degree $m=[(n-1) / 2]$, with arbitrary coefficients depending linearly on $b_{j}$. Hence, we fix our attention to study $M_{2, n}^{(1)}(\rho)$. We will prove by induction that $M_{2, n}^{(1)}(\rho)=q_{n-1}\left(\rho^{2}\right)$ for some polynomial $q_{n-1}$, of degree $n-1$, with coefficients depending quadratically on $a_{j}$. This proves the first statements. That is, the polynomial $M_{2, n}(\rho)=\rho p_{m}\left(\rho^{2}\right)+q_{n-1}\left(\rho^{2}\right)$ has $m+n+1$ monomials and, consequently from the Descartes signs' rule, the maximum number of positive zeros is $m+n$. The existence of such number of simple positive zeros is proved without the explicit computation of all their coefficients. Only the maximal degree term is necessary to be given. Because, at each step of the induction procedure, we add a complete polynomial in $\rho^{2}$ that has a new monomial of higher degree and it is independent with respect to the smaller degree monomials. Therefore, the last statement follows. In fact, the coefficient of maximal degree, $e_{2 n-2}$, takes different expressions depending on the parity of the degree of the perturbation. More concretely,

$e_{2 n-2}= \begin{cases}a_{n}^{+}\left(a_{n+1}^{+}+a_{n+1}^{-}\right) K_{n}, & \text { when } n \text { is even, } \\ a_{n+1}^{+}\left(a_{n}^{+}+a_{n}^{-}\right) K_{n}, & \text { when } n \text { is odd, }\end{cases}$

with positive constants $K_{n}$ satisfying the recursive relation

$K_{n}=\frac{n-1}{n+1} K_{n-2}-\frac{4}{\left(4(n-1)^{2}-1\right)(n+1)}$,

and $K_{1}=2, K_{2}=2 / 9$. The positiveness follows by induction proving that $K_{n}>1 /(n+1)^{2}>0$. We remark that the recurrence that defines $K_{n}$ does not change with the parity of $n$, but the initial values does.

We start writing system (24), with polar coordinates, in its equivalent piecewise one-form

$\begin{cases}d H+\varepsilon \omega_{1, n}^{+}+\varepsilon^{2} \omega_{2, n}^{+}=0 & \text { if } \theta \in[0, \pi), \\ d H+\varepsilon \omega_{1, n}^{-}+\varepsilon^{2} \omega_{2, n}^{-}=0 & \text { if } \theta \in[\pi, 2 \pi),\end{cases}$

where $H=r^{2} / 2,-\omega_{1, n}^{ \pm}$is defined in (25), and $-\omega_{2, n}^{ \pm}=$ $\alpha_{2, n}^{ \pm}(r, \theta) d r+\beta_{2, n}^{ \pm}(r, \theta) d \theta$, with

$\alpha_{2, n}^{ \pm}(r, \theta)=f_{n}^{ \pm}(r \cos \theta) \sin \theta=\sum_{j=0}^{n} b_{j}^{ \pm} r^{j} \cos ^{j} \theta \sin \theta$

$\beta_{2, n}^{ \pm}(r, \theta)=f_{n}^{ \pm}(r \cos \theta) r \cos \theta=\sum_{j=0}^{n} b_{j}^{ \pm} r^{j+1} \cos ^{j+1} \theta$.

The second Melnikov function, from Proposition 2.5, is given by

$M_{2, n}(\rho)=r_{2, n}^{+}(\pi, \rho)-r_{2, n}^{-}(-\pi, \rho)$.

Furthermore, from Proposition 2.3, we get

$$
\begin{aligned}
\rho r_{2, n}^{ \pm}(\theta, \rho)= & -\frac{1}{2} r_{1, n}^{ \pm}(\theta, \rho)^{2}+S_{2, n}^{ \pm}(\rho, \theta)+D_{1}\left(S_{1, n}^{ \pm}\right)(\rho, \theta) r_{1, n}^{ \pm}(\theta, \rho) \\
& +F_{2, n}^{ \pm}(\rho) \theta+\left(F_{1, n}^{ \pm}\right)^{\prime}(\rho) \int_{0}^{\theta} r_{1, n}^{ \pm}(\psi, \rho) d \psi,
\end{aligned}
$$

and $D_{1}\left(S_{1, n}^{ \pm}\right)(\rho, \theta)=\left.\frac{\partial S_{1, n}^{ \pm}(r, \theta)}{\partial r}\right|_{r=\rho}$. Here the functions $F_{1, n}^{ \pm}, S_{1, n}^{ \pm}$, and $r_{1, n}^{ \pm}$are defined in (27), (28), and (30), respectively, and the functions $F_{2, n}^{ \pm}$and $S_{2, n}^{ \pm}$are obtained applying Lemma 2.2 to the one-form

$-\Omega_{2, n}^{ \pm}=-\left(\omega_{2, n}^{ \pm}+h_{1, n}^{ \pm} \omega_{1, n}^{ \pm}\right)$.

From the hypothesis $\left(M_{1}\right)$ and the proof of Proposition 4.1 we have $r_{1, n}^{+}(\pi, \rho)=r_{1, n}^{-}(-\pi, \rho)$. Moreover, from (29), $S_{1, n}^{ \pm}(r, \pm \pi) \equiv 0$.
Hence, Eq. (33) writes as

$$
\begin{aligned}
M_{2, n}(\rho) & =\frac{1}{\rho}\left(\left(\left(F_{1, n}^{+}\right)^{\prime}(\rho) \int_{0}^{\pi} r_{1, n}^{+}(\psi, \rho) d \psi-\left(F_{1, n}^{-}\right)^{\prime}(\rho)\right.\right. \\
& \left.\times \int_{0}^{-\pi} r_{1, n}^{-}(\psi, \rho) d \psi\right) \\
& \left.+\left(F_{2, n}^{+}(\rho)+F_{2, n}^{-}(\rho)\right) \pi+\left(S_{2, n}^{+}(\rho, \pi)-S_{2, n}^{-}(\rho,-\pi)\right)\right) .
\end{aligned}
$$

From (34) it is clear that each $F_{2, n}^{ \pm}(\rho)$ and $S_{2, n}^{ \pm}(\rho)$ can be written as the sum of two terms, the one that depends linearly on the coefficients of the second order (computed from $\omega_{2, n}^{ \pm}$) and the ones that depend quadratically on the coefficients of the first order (computed from $\left.h_{1, n}^{ \pm} \omega_{1, n}^{ \pm}\right)$. We denote them by $F_{2, n+1}^{ \pm,(2)}(\rho)$, $S_{2, n+1}^{ \pm,(2)}(\rho), F_{2, n+1}^{ \pm,(1)}(\rho)$, and $S_{2, n+1}^{ \pm,(1)}(\rho)$, respectively. With this notation (35) writes as

$$
M_{2, n}(\rho)=M_{2, n}^{(2)}(\rho)+M_{2, n}^{(1)}(\rho)
$$

with

$$
\begin{aligned}
M_{2, n}^{(2)}(\rho)= & \left(F_{2, n}^{+,(2)}(\rho)+F_{2, n}^{-,(2)}(\rho)\right) \pi \\
& +\left(S_{2, n}^{+,(2)}(\rho, \pi)-S_{2, n}^{-,(2)}(\rho,-\pi)\right)
\end{aligned}
$$

and

$$
\begin{aligned}
M_{2, n}^{(1)}(\rho)= & \frac{1}{\rho}\left(\left(\left(F_{1, n}^{+}\right)^{\prime}(\rho) \int_{0}^{\pi} r_{1, n}^{+}(\psi, \rho) d \psi\right.\right. \\
& \left.-\left(F_{1, n}^{-}\right)^{\prime}(\rho) \int_{0}^{-\pi} r_{1, n}^{-}(\psi, \rho) d \psi\right) \\
& \left(F_{2, n}^{+,(1)}(\rho)+F_{2, n}^{-,(1)}(\rho)\right) \pi \\
& \left.+\left(S_{2, n}^{+,(1)}(\rho, \pi)-S_{2, n}^{-,(1)}(\rho,-\pi)\right)\right) .
\end{aligned}
$$

Also from Proposition 4.1, (36) writes as $M_{2, n}^{(2)}(\rho)=\rho p_{m}\left(\rho^{2}\right)$ where $p_{m}$ is the polynomial (31) changing $a_{i}$ by $b_{i}$. We recall that its degree is $m=[(n-1) / 2]$. So, we continue the proof showing that (37) writes as $M_{2, n}^{(1)}(\rho)=q_{n-1}\left(\rho^{2}\right)$, for some polynomial $q_{n-1}$ of degree $n-1$. As in the proof of Proposition 4.1, we will prove, by induction in $n$, that $M_{2, n}^{(1)}(\rho)$ function writes as

$M_{2, n+1}^{(1)}(\rho)=M_{2, n}^{(1)}(\rho)+P_{n}\left(\rho^{2}\right)$,

where the polynomial $P_{n}(\rho)$ has degree $n$. The proof will be done considering the three summands of Eq. (37) separately in the following parts (I), (II), and (III).

(I) The contribution of the first term to $M_{2, n+1}^{(1)}$ follows from the evaluation of the function $\left(F_{1, n+1}^{ \pm}\right)^{\prime}(\rho) \int_{0}^{\theta} r_{1, n+1}^{ \pm}(\psi, \rho) d \psi$ at $\theta=$ $\pm \pi$. Moreover, we will see that these expressions only provide even terms in $\rho$ for the polynomial $P_{n}(\rho)$.

From Eqs. (27) and (30) we get

$$
\begin{aligned}
\left(F_{1, n+1}^{ \pm}\right)^{\prime}(\rho) \int_{0}^{\theta} r_{1, n+1}^{ \pm}(\psi, \rho) d \psi \\
=\left(F_{1, n+1}^{ \pm}\right)^{\prime}(\rho) \int_{0}^{\theta}\left(r_{1, n}^{ \pm}(\psi, \rho)+a_{n+1}^{ \pm}\right. \\
\left.\quad \times\left(\mathcal{C}_{n+2}(\psi)+c_{n+2} \psi\right) \rho^{n+1}\right) d \psi \\
=\left(F_{1, n}^{ \pm}\right)^{\prime}(\rho) \int_{0}^{\theta} r_{1, n}^{ \pm}(\psi, \rho) d \psi+U_{1}^{ \pm}(\theta)+U_{2}^{ \pm}(\theta)+U_{3}^{ \pm}(\theta),
\end{aligned}
$$


where $F_{1, n}^{ \pm}(r)=\sum_{j=1}^{\left[\frac{n+1}{2}\right]} a_{2 j-1}^{ \pm} c_{2 j} r^{2 j}$ and

$U_{1}^{ \pm}(\theta)=\left(F_{1, n}^{ \pm}\right)^{\prime}(\rho) \int_{0}^{\theta} a_{n+1}^{ \pm}\left(\mathcal{C}_{n+2}(\psi)+c_{n+2} \psi\right) \rho^{n+1} d \psi$,

$U_{2}^{ \pm}(\theta)=a_{n+1}^{ \pm} c_{n+2}(n+2) \int_{0}^{\theta} r_{1, n}^{ \pm}(\psi, \rho) d \psi \rho^{n+1}$,

$U_{3}^{ \pm}(\theta)=a_{n+1}^{ \pm} c_{n+2}(n+2) \int_{0}^{\theta} a_{n+1}^{ \pm}\left(\mathcal{C}_{n+2}(\psi)+c_{n+2} \psi\right) \rho^{2 n+2} d \psi$.

Now, we will compute the evaluation of $U_{1}^{ \pm}(\theta), U_{2}^{ \pm}(\theta)$, and $U_{3}^{ \pm}(\theta)$ at $\theta= \pm \pi$ separately.

First, from Lemma 2.6, we have

$U_{1}^{ \pm}(\theta)=a_{n+1}^{ \pm}\left(\mathcal{C}_{0, n+2}(\theta)+\frac{c_{n+2}}{2} \theta^{2}\right) \sum_{j=1}^{\left[\frac{n+1}{2}\right]} 2 j a_{2 j-1}^{ \pm} c_{2 j} \rho^{2 j+n}$

and, due to hypothesis $\left(M_{1}\right)$ and $\mathcal{C}_{0, n+2}(\pi)=\mathcal{C}_{0, n+2}(-\pi)$,

$$
\begin{aligned}
\frac{U_{1}^{+}(\pi)-U_{1}^{-}(-\pi)}{\rho}= & \left(\mathcal{C}_{0, n+2}(\pi)+\frac{c_{n+2}}{2} \pi^{2}\right)\left(a_{n+1}^{+}+a_{n+1}^{-}\right) \\
& \times \sum_{j=1}^{\left[\frac{n+1}{2}\right]} 2 j a_{2 j-1}^{+} c_{2 j} \rho^{2 j+n-1} .
\end{aligned}
$$

This last expression is non identically zero when $n+1$ is even also due to hypothesis $\left(M_{1}\right)$ and it has only monomials of even degree. This is because, in this case, $c_{n+2}=0$. So, we write the right hand side of (39) as

$$
\left(a_{n+1}^{+}+a_{n+1}^{-}\right) \sum_{j=0}^{\left[\frac{n-1}{2}\right]} \eta_{j}^{(1)} a_{2 j+1}^{+} \rho^{2 j+n+1}
$$

for some real constants $\eta_{j}^{(1)}$, and moreover the term of higher degree in $\rho$ is

$(n+1) c_{n+1} a_{n}^{+}\left(a_{n+1}^{+}+a_{n+1}^{-}\right) \mathcal{C}_{0, n+2}(\pi) \rho^{2 n}$.

We remark that, in the above expression, $c_{n+1} \mathcal{C}_{0, n+2}(\pi) \neq 0$.

Second, from Lemma 2.6(h) and (30), we have

$U_{2}^{ \pm}(\theta)=a_{n+1}^{ \pm} c_{n+2}(n+2) \sum_{j=0}^{n} a_{j}^{ \pm}\left(\mathcal{C}_{0, j+1}(\theta)+\frac{c_{j+1}}{2} \theta^{2}\right) \rho^{n+j+1}$.

So, as $c_{n+2}\left(a_{n+1}^{+}+a_{n+1}^{-}\right)=0$ and $c_{2 j+1}=0$, for all $j$ and $n$, we get

$$
\begin{aligned}
\frac{U_{2}^{+}(\pi)-U_{2}^{-}(-\pi)}{\rho} \\
=c_{n+2}(n+2) \sum_{j=0}^{[n / 2]}\left(a_{n+1}^{+} a_{2 j}^{+}-a_{n+1}^{-} a_{2 j}^{-}\right) \\
\quad \times\left(\mathcal{C}_{0,2 j+1}(\pi)+\frac{c_{2 j+1}}{2} \pi^{2}\right) \rho^{2 j+n} \\
\quad+c_{n+2}(n+2)\left(a_{n+1}^{+}+a_{n+1}^{-}\right) \\
\quad \times \sum_{j=0}^{[n / 2]} a_{2 j+1}^{+}\left(\mathcal{C}_{0,2 j+2}(\pi)+\frac{c_{2 j+2}}{2} \pi^{2}\right) \rho^{2 j+1+n} \\
=c_{n+2}(n+2) \sum_{j=0}^{[n / 2]}\left(a_{n+1}^{+} a_{2 j}^{+}-a_{n+1}^{-} a_{2 j}^{-}\right) \mathcal{C}_{0,2 j+1}(\pi) \rho^{2 j+n} .
\end{aligned}
$$

We have used Lemmas 2.7(g), 2.6(b) and $\left(M_{1}\right)$. The expression (42) is non identically zero only when $n$ is even. Consequently, the last expression in (42) writes as

$a_{n+1}^{+} \sum_{j=0}^{[n / 2]} \eta_{j}^{(2)}\left(a_{2 j}^{+}+a_{2 j}^{-}\right) \rho^{2 j+n}$

for some real constants $\eta_{j}^{(2)}$. Clearly it has only monomials of even degree and the term of higher degree in $\rho$ is

$(n+2) c_{n+2} a_{n+1}^{+}\left(a_{n}^{+}+a_{n}^{-}\right) \mathcal{C}_{0, n+1}(\pi) \rho^{2 n}$.

We also remark that, in the above expression, $c_{n+2} \mathcal{C}_{0, n+1}(\pi) \neq 0$.

Third, using also Lemma 2.6(h), we have

$U_{3}^{ \pm}(\theta)=\left(a_{n+1}^{ \pm}\right)^{2} c_{n+2}(n+2)\left(\mathcal{C}_{0, n+2}(\theta)+c_{n+2} \frac{\theta^{2}}{2}\right) \rho^{2 n+2}$

and, consequently,

$$
\begin{aligned}
\frac{U_{3}^{+}(\pi)-U_{3}^{-}(-\pi)}{\rho}= & (n+2) c_{n+2}\left(\mathcal{C}_{0, n+2}(\pi)+c_{n+2} \frac{\pi^{2}}{2}\right) \\
& \times\left(\left(a_{n+1}^{+}\right)^{2}-\left(a_{n+1}^{-}\right)^{2}\right) \rho^{2 n+2}=0 .
\end{aligned}
$$

Because, when $n$ is even, by hypothesis $\left(M_{1}\right), a_{n+1}^{+}+a_{n+1}^{-}=0$ and when $n$ is odd $c_{n+2}=0$.

(II) The contribution of the second term to $M_{2, n+1}^{(1)}$ in (35) follows from the computation of $F_{2, n+1}^{ \pm,(1)}(\rho)$. In fact we will prove that both functions vanish identically.

From the second summand of (34) we have

$F_{2, n+1}^{ \pm,(1)}(\rho)=\frac{1}{2 \pi} \int_{0}^{2 \pi} h_{1, n+1}^{ \pm}(\rho, \theta) \beta_{1, n+1}^{ \pm}(\rho, \theta) d \theta$.

Straightforward computations show, from the definition of $h_{1}$, see Lemma 2.2, and Eqs. (25) and (28), that $h_{1,0}^{ \pm}(r, \theta)=0$ and

$h_{1, n+1}^{ \pm}(r, \theta)=h_{1, n}^{ \pm}(r, \theta)-a_{n+1}^{ \pm}(n+1) \mathcal{C}_{n}(\theta) r^{n}$.

In particular, by induction,

$h_{1, n+1}^{ \pm}(r, \theta)=-\sum_{j=0}^{n} a_{j+1}^{ \pm}(j+1) \mathcal{C}_{j}(\theta) r^{j}$

Finally, also from Lemma 2.6(d) and Eq. (25), it follows that (46) is zero.

(III) The contribution of the third term to $M_{2, n+1}^{(1)}$ in (35) follows from the evaluation of the functions $S_{2, n+1}^{ \pm,(1)}(\rho, \theta)$ at $\theta= \pm \pi$. Moreover, we will see that these expressions only provide even terms in $\rho$ for the polynomial $P_{n}(\rho)$.

From the second summand of (34) and the fact that $F_{2, n+1}^{ \pm,(1)}(\rho) \equiv$ 0 , we have

$$
\begin{aligned}
S_{2, n+1}^{ \pm,(1)}(\rho, \theta)= & \int_{0}^{\theta} h_{1, n+1}^{ \pm}(\rho, \psi) \beta_{1, n+1}^{ \pm}(\rho, \psi) d \psi \\
= & \int_{0}^{\theta}\left(h_{1, n}^{ \pm}(\rho, \psi)-a_{n+1}^{ \pm}(n+1) \mathcal{C}_{n}(\psi) \rho^{n}\right) \\
& \times\left(\beta_{1, n}^{ \pm}(\rho, \psi)+a_{n+1}^{ \pm} \rho^{n+2} \cos ^{n+2} \psi\right) d \psi \\
= & S_{2, n}^{ \pm,(1)}(\rho, \psi)+V_{1}^{ \pm}(\theta)+V_{2}^{ \pm}(\theta)+V_{3}^{ \pm}(\theta),
\end{aligned}
$$

where

$$
\begin{aligned}
& V_{1}^{ \pm}(\theta)=-a_{n+1}^{ \pm}(n+1) \rho^{n} \int_{0}^{\theta} \beta_{1, n}^{ \pm}(\rho, \psi) \mathcal{C}_{n}(\psi) d \psi, \\
& V_{2}^{ \pm}(\theta)=a_{n+1}^{ \pm} \rho^{n+2} \int_{0}^{\theta} \cos ^{n+2} \psi h_{1, n}^{ \pm}(\rho, \psi) d \psi, \\
& V_{3}^{ \pm}(\theta)=-\left(a_{n+1}^{ \pm}\right)^{2}(n+1) \rho^{2 n+2} \int_{0}^{\theta} \cos ^{n+2} \psi \mathcal{C}_{n}(\psi) d \psi
\end{aligned}
$$

Now we will compute the evaluation of $V_{1}^{ \pm}(\theta), V_{2}^{ \pm}(\theta)$, and $V_{3}^{ \pm}(\theta)$ at $\theta= \pm \pi$ separately. 
First, from Lemma 2.6(h) and the definition of $\beta_{1, n}^{ \pm}$in (25), we have that

$V_{1}^{ \pm}(\theta)=-a_{n+1}^{ \pm}(n+1) \sum_{j=0}^{n} a_{j}^{ \pm} \mathcal{C}_{j+1, n}(\theta) \rho^{n+j+1}$.

So, from Lemma 2.7,

$$
\begin{aligned}
\frac{V_{1}^{+}(\pi)-V_{1}^{-}(-\pi)}{\rho}= & (n+1) \sum_{j=0}^{n}\left(a_{n+1}^{-} a_{j}^{-}-a_{n+1}^{+} a_{j}^{+}\right) \\
& \times \mathcal{C}_{j+1, n}(\pi) \rho^{n+j} .
\end{aligned}
$$

The above polynomial has only monomials of even degree because, when $n+j+1$ is even, the corresponding $\mathcal{C}_{j+1, n}(\pi)=0$, see also Lemma 2.7(g). Moreover, the term of higher degree writes as

$(n+1)\left(a_{n+1}^{-} a_{n}^{-}-a_{n+1}^{+} a_{n}^{+}\right) \mathcal{C}_{n+1, n}(\pi) \rho^{2 n}$

and the right hand side of (47) writes as (40) or (43), changing the coefficients $\eta_{j}^{(1)}$ and $\eta_{j}^{(2)}$, when $n$ is odd or even, respectively. The maximal degree term is different from zero because $\mathcal{C}_{n+1, n}(\pi) \neq 0$.

Second, from Lemma 2.6(h), we have that

$$
\begin{aligned}
V_{2}^{ \pm}(\theta) & =a_{n+1}^{ \pm} \rho^{n+2} \int_{0}^{\theta} \cos ^{n+2} \psi\left(-\sum_{j=0}^{n-1} a_{j+1}^{ \pm}(j+1) \mathcal{C}_{j}(\psi) \rho^{j}\right) d \psi \\
& =-a_{n+1}^{ \pm} \sum_{j=0}^{n-1} a_{j+1}^{ \pm}(j+1) \mathcal{C}_{n+2, j}(\theta) \rho^{n+j+2} .
\end{aligned}
$$

So, again from Lemma 2.7, we have that

$$
\begin{aligned}
\frac{V_{2}^{+}(\pi)-V_{2}^{-}(-\pi)}{\rho}= & \sum_{j=0}^{n-1}\left(a_{n+1}^{-} a_{j+1}^{-}-a_{n+1}^{+} a_{j+1}^{+}\right)(j+1) \\
& \times \mathcal{C}_{n+2, j}(\pi) \rho^{n+j+1} .
\end{aligned}
$$

The above polynomial has only monomials of even degree because when $n+j+2$ is even the corresponding $\mathcal{C}_{n+2, j}(\pi)=0$, see also Lemma 2.7(g). Moreover, the term of higher degree writes as

$n\left(a_{n+1}^{-} a_{n}^{-}-a_{n+1}^{+} a_{n}^{+}\right) \mathcal{C}_{n+2, n-1}(\pi) \rho^{2 n}$

and, as before, the right hand side of (49) writes as (40) or (43), changing the coefficients $\eta_{j}^{(1)}$ and $\eta_{j}^{(2)}$, when $n$ is odd or even, respectively. Here, also the maximal degree term is different from zero because $\mathcal{C}_{n+2, n-1}(\pi) \neq 0$.

Third, from Lemma 2.6(h), we have that

$$
\begin{aligned}
V_{3}^{ \pm}(\theta) & =-\left(a_{n+1}^{ \pm}\right)^{2}(n+1) \rho^{2 n+2} \int_{0}^{\theta} \cos ^{n+2} \psi \mathcal{C}_{n}(\psi) d \psi \\
& =-\left(a_{n+1}^{ \pm}\right)^{2}(n+1) \rho^{2 n+2} \mathcal{C}_{n+2, n}(\theta) .
\end{aligned}
$$

So, using that $\mathcal{C}_{n+2, n}(\pi)=\mathcal{C}_{n+2, n}(-\pi)=0$,

$$
\frac{V_{3}^{+}(\pi)-V_{3}^{-}(-\pi)}{\rho}=0 \text {. }
$$

From all the computations done above we have proved, by induction, that (37) writes as $M_{2, n}^{(1)}(\rho)=q_{n-1}\left(\rho^{2}\right)$ for some polynomial $q_{n-1}$ of degree $n-1$. This is due to the fact that the polynomial $P_{n}\left(\rho^{2}\right)$, in (38), follows adding the polynomials (39), (42), (45), (47), (49), and (51), all of them having only monomials of even degree. More concretely, it writes in the form (40) or (43) when $n$ is odd or even, respectively. Moreover, the coefficient of maximal degree term, $\rho^{2 n}$, is obtained adding (41), (44), (48), and (50). In what follows we compute it explicitly because it is necessary to conclude the proof, showing also that it does not vanish. We remark that in both expressions, $P_{n}\left(\rho^{2}\right)$ and $\rho^{2 n}$, the $n$ refers to the perturbation of degree $n+1$, because of the induction procedure.
Next, we will write, to simplify the computations, the coefficient of maximal degree corresponding to $M_{2, n+1}(\rho)$ or $M_{2, n+1}^{(1)}(\rho)$, because they coincide.

When $n$ is odd, we have that the coefficient of maximal degree is $a_{n}^{+}\left(a_{n+1}^{+}+a_{n+1}^{-}\right) \widetilde{K}_{n}$ with

$\widetilde{K}_{n}=(n+1) c_{n+1} \mathcal{C}_{0, n+2}(\pi)-(n+1) \mathcal{C}_{n+1, n}(\pi)-n \mathcal{C}_{n+2, n-1}(\pi)$.

This last expression can be simplified using properties (f) and (g) of Lemma 2.7. First, in the second and third summands, writing it as

$\widetilde{K}_{n}=\frac{4(n+1)}{(2 n+1)(n+2)}-\frac{n}{n+2} \mathcal{C}_{n-1, n}(\pi)$,

and then, in the second summand of the above expression, to write a recurrent expression for the coefficient of maximal degree,

$\widetilde{K}_{n}=\frac{n}{n+2} \widetilde{K}_{n-2}-\frac{4}{\left(4 n^{2}-1\right)(n+2)}$.

The first value follows by direct computation and we obtain $\widetilde{K}_{1}=$ $2 / 9$. All the coefficients are positive because we can prove, by induction, that $\widetilde{K}_{n}>1 /(n+2)^{2}>0$. When $n$ is even, the coefficient of maximal degree writes now as $a_{n+1}^{+}\left(a_{n}^{+}+a_{n}^{-}\right) \widetilde{K}_{n}$ with

$\widetilde{K}_{n}=(n+2) c_{n+2} \mathcal{C}_{0, n+1}(\pi)-(n+1) \mathcal{C}_{n+1, n}(\pi)-n \mathcal{C}_{n+2, n-1}(\pi)$.

Using similarly Lemma 2.7 as previously we have the same recurrence relation (52). But starting at $\widetilde{K}_{0}=2$. Clearly, $\widetilde{K}_{n}$ is also positive because the same lower bound applies.

We remark that the expression for (32) follows from (52) because $\widetilde{K}_{n-1}=K_{n}$. Consequently, $K_{n}>1 /(n+1)^{2}>0$.

The last statement of the theorem follows writing the second Melnikov function $M_{2, n}(\rho)$ as $\rho p_{m}\left(\rho^{2}\right)+q_{n-1}\left(\rho^{2}\right)$, for $a_{2 k}^{-}=0$ when $k=0, \ldots,[n / 2]$ and $b_{2 k+1}^{-}=0$ when $k=0, \ldots m$. As we have mentioned before, the polynomial $p_{m}(\rho)$ writes as (31) changing $a_{2 k+1}^{+}$to $b_{2 k+1}^{+}$and $a_{2 k+1}^{-}$to 0 . The polynomial $q_{n-1}(\rho)$ is obtained by induction, using alternatively (40) and (43). So, we have

$M_{2, n}(\rho)=\sum_{k=0}^{m} \zeta_{k} b_{2 k+1}^{+} \rho^{2 k+1}+\sum_{\ell=0}^{n-1}\left(\sum_{\substack{i+j=2 \ell+1 \\ i, j<n}} \xi_{i j} a_{i}^{+} a_{j}^{+}\right) \rho^{2 \ell}$,

where $\zeta_{k} \neq 0$ and $\xi_{\ell, \ell+1}=K_{\ell} \neq 0$, due to (31) and (32), respectively. This last expression, expanding the sums, writes

$$
\begin{aligned}
M_{2, n}(\rho)= & K_{0} a_{0}^{+} a_{1}^{+}+\zeta_{0} b_{1}^{+} \rho+\left(\xi_{03} a_{0}^{+} a_{3}^{+}+K_{1} a_{1}^{+} a_{2}^{+}\right) \rho^{2}+\zeta_{1} b_{3}^{+} \rho^{3} \\
& +\left(\xi_{05} a_{0}^{+} a_{5}^{+}+\xi_{14} a_{1}^{+} a_{4}^{+}+K_{2} a_{2}^{+} a_{3}^{+}\right) \rho^{4}+\cdots .
\end{aligned}
$$

It remains to prove the existence of parameters $a_{k}^{+}$and $b_{k}^{+}$such that (53) has exactly $m+n$ simple zeros. They appear bifurcating from infinity according to the following procedure. We start choosing all the parameters equals zero except $a_{0}^{+}=a_{1}^{+}=1$ and we continue adding the rest of parameters one by one, with a specific order, $b_{1}^{+}, a_{2}^{+}, b_{3}^{+}, a_{3}^{+}, \ldots, b_{m}^{+}, a_{m}^{+}, a_{m+1}^{+}, \ldots, a_{n-1}^{+}, a_{n}^{+}$, and alternating signs. That is adding only one monomial of higher degree in each step. The first simple zero is obtained taking any $b_{1}^{+}<0$. The second appears, from infinity, choosing $a_{2}^{+}>0$ small enough, because at this step $a_{3}^{+}=0$. The third bifurcates also from infinity adding $b_{3}^{+}<0$ small enough. At the fourth step, adding $a_{3}^{+}>0$ also small enough, the previous zeros remain, by continuity, close to the ones appeared in the previous step, but the coefficient of $\rho^{2}$ changes slightly. The proof ends when all the coefficients of (53) have been added.

From the last proof, it is clear that $M_{2, n}$, see (53), has exactly $m+n$ positive simple zeros and no more. Moreover, only $K_{\ell}$ and $\zeta_{k}$ should be different from zero. The next example shows the explicit 
expression for $M_{2}$ when $n=6$,

$$
\begin{aligned}
M_{2,6}(\rho)= & \frac{1774}{24255} a_{5}^{+} a_{6}^{+} \rho^{10}+\left(-\frac{702}{1225} a_{3}^{+} a_{6}^{+}+\frac{578}{945} a_{4}^{+} a_{5}^{+}\right) \rho^{8} \\
& +\left(-\frac{1426}{735} a_{1}^{+} a_{6}^{+}+\frac{26}{21} a_{2}^{+} a_{5}^{+}+\frac{58}{525} a_{3}^{+} a_{4}^{+}\right) \rho^{6} \\
& +\frac{5 \pi}{16} b_{5}^{+} \rho^{5} \\
& +\left(2 a_{0}^{+} a_{5}^{+}-\frac{74}{75} a_{1}^{+} a_{4}^{+}+\frac{14}{15} a_{2}^{+} a_{3}^{+}\right) \rho^{4}+\frac{3 \pi}{8} b_{3}^{+} \rho^{3} \\
& +\left(2 a_{0}^{+} a_{3}^{+}+\frac{2}{9} a_{1}^{+} a_{2}^{+}\right) \rho^{2}+\frac{\pi}{2} b_{1}^{+} \rho+2 a_{1}^{+} a_{0}^{+} .
\end{aligned}
$$

Then, it seems numerically that the eight positive simple zeros that the above polynomial can have, can be located arbitrarily in the positive real line. For example, if we fix the values $a_{0}^{+}=1, a_{1}^{+}=$ $1, a_{2}^{+}=0.02154486, a_{3}^{+}=2.6966906, a_{4}^{+}=-0.1355754$. $10^{-3}, a_{5}^{+}=0.3935304, a_{6}^{+}=0.4103265 \cdot 10^{-5}, b_{1}^{+}=$ $-3.351348, b_{3}^{+}=-2.4176393, b_{5}^{+}=-0.1400563$, then $M_{6}$ has only 8 real zeros at $\rho=1,2,3, \ldots, 7,8$.

Alternatively, choosing $a_{n-1}^{+}=a_{n}^{+}=1$, the zeros can be obtained bifurcating from the origin reordering in an adequate way the perturbation parameters.

\section{Perturbations of any order for some classic Liénard families}

In the previous section we have seen that the study of the second Melnikov function for classical Liénard equations with a horizontal discontinuity line is more difficult than the first order study. A higher order analysis for perturbations of degree $n$ can only be done adding some extra hypotheses or changing the slope of the discontinuity line. This is what we do in Theorems 1.3 and 1.4. In the first result we assume some symmetries on the perturbations and in the second we change the discontinuity line to the vertical axis. Their proofs follow directly from the next two propositions.

\section{Proposition 5.1.}

(a) When $f_{i}^{ \pm}$are even polynomials, system (3) has a center at the origin for every $\varepsilon$. Moreover, all the Melnikov functions vanish.

(b) When $f_{i}^{ \pm}$are odd polynomials, the Melnikov function of order $N, M_{N}$, associated to system (3) has at most $(n-1) / 2$ zeros. Moreover, there exist polynomials $f_{i}^{ \pm}$such that the zeros are realized as simple ones.

Now we consider system (4). Under the change of coordinates $\{x=y, y=x\}$ it becomes

$\left(x^{\prime}, y^{\prime}\right)=\left(y,-x+\sum_{i=1}^{N} \varepsilon^{i} f_{i}^{ \pm}(y)\right)$,

where $f_{i}^{ \pm}$are polynomials of degree $n$ defined now in $\Sigma_{0}^{ \pm}=$ $\left\{(x, y) \in \mathbb{R}^{2}: \pm y \geq 0\right\}$. Observe that, for this new system, the discontinuity set is the $x$-axis.

Proposition 5.2. All the Melnikov functions associated to system (54) have at most $n$ zeros. Moreover, there exist polynomials $f_{i}^{ \pm}$such that all the zeros are realized as simple ones.

Remark 5.3. In the above results every Melnikov function has exactly the same form. In fact all the higher order studies coincide with the first one. We can say that the Melnikov function stabilizes in the first step. Like in the Liénard smooth case, see [8].

Proof of Proposition 5.1. (a) Each system defined by (3), assuming the even property in $f^{ \pm}$, is symmetric with respect the $y$ axis. In fact it is invariant via the change of variables $(x, y, t) \rightarrow(-x, y,-t)$. Hence, the system is globally invariant with respect to this change of variables and, consequently, the respective half-return maps are the identity ones for every $\varepsilon: \Pi^{+}(\rho)=\Pi^{-}(\rho)=-\rho$. Then the Melnikov functions vanish identically for each order and the proof finishes.

(b) First, system (3), assuming the odd symmetry in $f^{ \pm}$and writing $f_{i}^{ \pm}(x)=x \hat{f}_{i}^{ \pm}\left(x^{2}\right)=x \sum_{j=0}^{n} a_{2 j+1, i}^{ \pm}\left(x^{2}\right)^{j}$ where $a_{2 j+1, i} \in \mathbb{R}$, can be expressed as

$$
\left\{\begin{array}{l}
d H+\sum_{i=1}^{N} \varepsilon^{i} \omega_{i}^{+}=0, \quad \text { if } \theta \in[0, \pi), \\
d H+\sum_{i=1}^{N} \varepsilon^{i} \omega_{i}^{-}=0, \quad \text { if } \theta \in[\pi, 2 \pi),
\end{array}\right.
$$

where $-\omega_{i}^{ \pm}=\alpha_{i}^{ \pm}(r, \theta) d r+\beta_{i}^{ \pm}(r, \theta)$ with

$$
\begin{gathered}
\alpha_{i}^{ \pm}(r, \theta)=\sum_{j=0}^{n} a_{2 j+1, i}^{ \pm} r^{2 j+1} \cos ^{2 j+1} \theta \sin \theta \quad \text { and } \\
\beta_{i}^{ \pm}(r, \theta)=\sum_{j=0}^{n} a_{2 j+1, i}^{ \pm} r^{2 j+2} \cos ^{2 j+2} \theta .
\end{gathered}
$$

In order to find the Melnikov functions of order $i, M_{i}$, we will write $r^{ \pm}(\theta, \varepsilon, \rho)$, the solution of (55), as

$r^{ \pm}(\theta, \varepsilon, \rho)=\sum_{i=0}^{N} \varepsilon^{i} r_{i}^{ \pm}(\theta, \rho)$.

Consequently, $M_{i}(\rho)=r_{i}(\pi, \rho)-r_{i}^{-}(-\pi, \rho)$.

The first Melnikov function is given by $M_{1}=r_{1}^{+}(\pi)-r_{1}^{-}(-\pi)$ where, from Proposition 2.3, the functions $r_{1}^{ \pm}(\theta):=r_{1}^{ \pm}(\theta, \rho)$ satisfy

$\rho r_{1}^{ \pm}(\theta)=F_{1}^{ \pm}(\rho) \theta+S_{1}^{ \pm}(\rho, \theta)$,

and $F_{1}^{ \pm}, S_{1}^{ \pm}$are obtained from the one-forms $-\Omega_{1}^{ \pm}$where $h_{0}^{ \pm}=1$ and

$-\Omega_{1}^{ \pm}=-\omega_{1}^{ \pm} h_{0}^{ \pm}=\alpha_{1}^{ \pm}(r, \theta) d r+\beta_{1}^{ \pm}(r, \theta) d \theta$.

With this notation, the functions $F_{1}^{ \pm}$and $S_{1}^{ \pm}$are given by the expressions

$$
\begin{aligned}
& F_{1}^{ \pm}(r)=\frac{1}{2 \pi} \int_{0}^{2 \pi} \beta_{1}^{ \pm}(r, \theta) d \theta \text { and } \\
& S_{1}^{ \pm}(r, \theta)=\int_{0}^{\theta} \beta_{1}^{ \pm}(r, \psi) d \psi-F_{1}^{ \pm}(r) \theta .
\end{aligned}
$$

So we get

$$
\begin{aligned}
& F_{1}^{ \pm}(r)=\sum_{j=0}^{n} a_{2 j+1,1}^{ \pm} c_{2 j+2} r^{2 j+2} \text { and } \\
& S_{1}^{ \pm}(r, \theta)=\sum_{j=0}^{n} a_{2 j+1,1}^{ \pm} \mathcal{C}_{2 j+2}(\theta) r^{2 j+2} .
\end{aligned}
$$

where, $c_{2 j+2}$ and $\mathcal{C}_{2 j+2}$ are given in Lemma 2.6 and the function $\mathcal{C}_{2 j+2}$ has the form $\sin \theta p_{2 j+1}(\cos \theta)$ with $p_{2 j+1}$ a polynomial of degree $2 j+1$ in $\cos \theta$. Moreover,

$h_{1}^{ \pm}(r, \theta)=\frac{1}{r}\left(\alpha_{1}^{ \pm}(r, \theta)-\frac{\partial S_{1}^{ \pm}(r, \theta)}{\partial r}\right)$.

Observe that, from Eqs. (57) and (58), we have $S_{1}^{ \pm}(r,-\theta)=-S_{1}^{ \pm}(r, \theta)$ and $r_{1}^{ \pm}(-\theta)=-r_{1}^{ \pm}(\theta)$. 
And, from the fact that $S_{1}^{+}(\rho, \pi)=S_{1}^{-}(\rho,-\pi)=0$, it follows from Eq. (57) that

$$
\begin{aligned}
M_{1}(\rho)= & r_{1}^{+}(\pi)-r_{1}^{-}(-\pi)=\frac{F_{1}^{+}(\rho)+F_{1}^{-}(\rho)}{\rho} \pi \\
& +\frac{S_{1}^{+}(\rho, \pi)-S_{1}^{-}(\rho,-\pi)}{\rho}=\frac{F_{1}^{+}(\rho)+F_{1}^{-}(\rho)}{\rho} \pi .
\end{aligned}
$$

Suppose that the first Melnikov function vanishes identically, that is, $M_{1}(\rho) \equiv 0$. Then $F_{1}^{+}(\rho)=-F_{1}^{-}(\rho)$ and from (58) we obtain $a_{2 j+1,1}^{-}=-a_{2 j+1,1}^{+}, j=1, \ldots, n$. In particular, from Eqs. (56), (57), and (58) we get

$$
\begin{aligned}
\alpha_{1}^{-}(r, \theta) & =-\alpha_{1}^{+}(r, \theta), & \beta_{1}^{-}(r, \theta) & =-\beta_{1}^{+}(r, \theta), \\
S_{1}^{+}(r, \theta) & =-S_{1}^{-}(r, \theta), & r_{1}^{-}(\theta) & =-r_{1}^{+}(\theta) .
\end{aligned}
$$

Also as $\alpha_{1}^{ \pm}$and $S_{1}^{ \pm}$are odd functions with respect to the variable $\theta$, it follows that

$h_{1}^{ \pm}(r,-\theta)=-h_{1}^{ \pm}(r, \theta)$.

From the equalities above and the expressions (56) and (59) we can write

$$
F_{1}^{+}(\rho)+F_{1}^{-}(\rho)=0, \quad S_{1}^{+}(\rho, \theta)-S_{1}^{-}(\rho,-\theta)=0,
$$

$h_{1}^{+}(\rho, \theta)-h_{1}^{-}(\rho,-\theta)=0, \quad \alpha_{1}^{+}(\rho, \theta)-\alpha_{1}^{-}(\rho,-\theta)=0$,

$$
\beta_{1}^{+}(\rho, \theta)+\beta_{1}^{-}(\rho,-\theta)=0, \quad r_{1}^{+}(\theta)-r_{1}^{-}(-\theta)=0 .
$$

Now taking the $\varepsilon^{2}$-terms in the series expansion of Eq. (8) of Proposition 2.3 we get

$$
\begin{aligned}
\rho r_{2}^{ \pm}(\theta)= & -\frac{1}{2}\left(r_{1}^{ \pm}(\theta)\right)^{2}+\mathcal{O}_{\varepsilon}\left(\int_{0}^{\theta} F_{1}^{ \pm}\left(\rho+\varepsilon r_{1}^{ \pm}(\psi)\right) d \psi\right. \\
& \left.+S_{1}^{ \pm}\left(\rho+\varepsilon r_{1}^{ \pm}(\theta), \theta\right)\right) \\
& +F_{2}^{ \pm}(\rho) \theta+S_{2}^{ \pm}(\rho, \theta),
\end{aligned}
$$

where $\mathcal{O}_{\varepsilon}(\cdot)$ denotes the order $\varepsilon$ terms of $(\cdot)$.

The second Melnikov function is given by $M_{2}=r_{2}^{+}(\pi)-r_{2}^{-}(-\pi)$ and, from conditions (60), we have

$$
\begin{aligned}
\int_{0}^{-\theta} F_{1}^{-}\left(\rho+\varepsilon r_{1}^{-}(\psi)\right) d \psi & =-\int_{0}^{\theta} F_{1}^{-}\left(\rho+\varepsilon r_{1}^{-}(-\psi)\right) d \psi \\
& =-\int_{0}^{\theta} F_{1}^{-}\left(\rho+\varepsilon r_{1}^{+}(\psi)\right) d \psi \\
& =\int_{0}^{\theta} F_{1}^{+}\left(\rho+\varepsilon r_{1}^{+}(\psi)\right) d \psi
\end{aligned}
$$

Also, $S_{1}^{-}\left(\rho+\varepsilon r_{1}^{-}(-\theta),-\theta\right)=-S_{1}^{-}\left(\rho+\varepsilon r_{1}^{-}(-\theta), \theta\right)=-S_{1}^{-}(\rho+$ $\left.\varepsilon r_{1}^{+}(\theta), \theta\right)$ that implies

$$
\begin{aligned}
& S_{1}^{+}\left(\rho+\varepsilon r_{1}^{+}(\theta), \theta\right)-S_{1}^{-}\left(\rho+\varepsilon r_{1}^{-}(-\theta),-\theta\right) \\
& \quad=S_{1}^{+}\left(\rho+\varepsilon r_{1}^{+}(\theta), \theta\right)+S_{1}^{-}\left(\rho+\varepsilon r_{1}^{+}(\theta), \theta\right)=0 .
\end{aligned}
$$

Next, from (61), we write

$$
\begin{aligned}
r_{2}^{+}(\theta)-r_{2}^{-}(-\theta)= & \frac{F_{2}^{+}(\rho)+F_{2}^{-}(\rho)}{\rho} \theta \\
& +\frac{S_{2}^{+}(\rho, \theta)-S_{2}^{-}(\rho,-\theta)}{\rho}
\end{aligned}
$$

where the functions $F_{2}^{ \pm}, S_{2}^{ \pm}$and $h_{2}^{ \pm}$are obtained in a similar way as the case $i=1$ but now using the one-forms

$$
-\Omega_{2}^{ \pm}=-\omega_{2}^{ \pm}-h_{1}^{ \pm} \omega_{1}^{ \pm}=\hat{\alpha}_{2}^{ \pm}(r, \theta) d r+\hat{\beta}_{2}^{ \pm}(r, \theta) d \theta,
$$

with

$$
\begin{aligned}
\hat{\alpha}_{2}^{ \pm}(r, \theta) & =\alpha_{2}^{ \pm}(r, \theta)+h_{1}^{ \pm}(r, \theta) \alpha_{1}^{ \pm}(r, \theta) \\
& =\alpha_{2}^{ \pm}(r, \theta)+\alpha_{2}^{ \pm,(1)}(r, \theta), \\
\hat{\beta}_{2}^{ \pm}(r, \theta) & =\beta_{2}^{ \pm}(r, \theta)+h_{1}^{ \pm}(r, \theta) \beta_{1}^{ \pm}(r, \theta) \\
& =\beta_{2}^{ \pm}(r, \theta)+\beta_{2}^{ \pm,(1)}(r, \theta) .
\end{aligned}
$$

So, we have

$$
\begin{gathered}
F_{2}^{ \pm}(\rho)=F_{2}^{ \pm,(2)}(\rho)+F_{2}^{ \pm,(1)}(\rho), \quad \text { and } \\
S_{2}^{ \pm}(\rho, \theta)=S_{2}^{ \pm,(2)}(\rho, \theta)+S_{2}^{ \pm,(1)}(\rho, \theta),
\end{gathered}
$$

where $F_{2}^{ \pm,(2)}, S_{2}^{ \pm,(2)}$ depend on the coefficients of order $\varepsilon^{2}$ of the one-forms (55) and are given by

$$
\begin{aligned}
F_{2}^{ \pm,(2)}(r) & =\frac{1}{2 \pi} \int_{0}^{2 \pi} \beta_{2}^{ \pm}(r, \theta) d \theta=\sum_{j=0}^{n} a_{2 j+1,2}^{ \pm} c_{2 j+2} r^{2 j+1}, \\
S_{2}^{ \pm,(2)}(r, \theta) & =\int_{0}^{\theta} \beta_{2}^{ \pm}(r, \psi) d \psi-F_{2}^{ \pm,(2)}(r) \theta \\
& =\sum_{j=0}^{n} a_{2 j+1,2}^{ \pm} \mathcal{C}_{2 j+2}(\theta) r^{2 j+2},
\end{aligned}
$$

where $\mathcal{C}_{2 j+2}$ is given in Eq. (58). The functions $F_{2}^{ \pm,(1)}, S_{2}^{ \pm,(1)}$ depend on the coefficients of order $\varepsilon$ of the one-forms (55) and are given by

$$
\begin{aligned}
F_{2}^{ \pm,(1)}(r) & =\frac{1}{2 \pi} \int_{0}^{2 \pi} \beta_{2}^{ \pm,(1)}(r, \theta) d \theta \\
S_{2}^{ \pm,(1)}(r, \theta) & =\int_{0}^{\theta} \beta_{2}^{ \pm,(1)}(r, \psi) d \psi-F_{2}^{ \pm,(1)}(r) \theta .
\end{aligned}
$$

Moreover, from the fact that $\beta_{2}^{+,(1)}(r, \theta)=-\beta_{2}^{-,(1)}(r, \theta)$, these functions satisfy

$F_{2}^{+,(1)}(r)=-F_{2}^{-,(1)}(r), \quad$ and $\quad S_{2}^{+,(1)}(r, \theta)=-S_{2}^{-,(1)}(r, \theta)$.

Now it follows from Eqs. (60) and (65) that

$$
\begin{aligned}
& S_{2}^{+,(1)}(r, \theta)-S_{2}^{-,(1)}(r,-\theta)=\int_{0}^{\theta} \beta_{2}^{+,(1)}(r, \psi) d \psi \\
& \quad-\int_{0}^{-\theta} \beta_{2}^{-,(1)}(r, \psi) d \psi \\
& =\int_{0}^{\theta}\left(\beta_{2}^{+,(1)}(r, \psi)+\beta_{2}^{-,(1)}(r,-\psi)\right) d \psi \\
& =\int_{0}^{\theta}\left(\beta_{2}^{+,(1)}(r, \psi)+h_{1}^{-}(r,-\psi) \beta_{1}^{-}(r,-\psi)\right) d \psi \\
& =\int_{0}^{\theta}\left(\beta_{2}^{+,(1)}(r, \psi)-h_{1}^{+}(r, \psi) \beta_{1}^{+}(r, \psi)\right) d \psi \\
& =\int_{0}^{\theta}\left(\beta_{2}^{+,(1)}(r, \psi)-\beta_{2}^{+,(1)}(r, \psi)\right)=0 .
\end{aligned}
$$

So we obtain from Eq. (62) the difference

$$
\begin{aligned}
r_{2}^{+}(\theta)-r_{2}^{-}(-\theta)= & \frac{F_{2}^{+}(\rho)+F_{2}^{-}(\rho)}{\rho} \theta+\frac{S_{2}^{+}(\rho, \theta)-S_{2}^{-}(\rho, \theta)}{\rho} \\
= & \frac{F_{2}^{+,(2)}(\rho)+F_{2}^{-,(2)}(\rho)}{\rho} \theta \\
& +\frac{S_{2}^{+,(2)}(\rho, \theta)-S_{2}^{-,(2)}(\rho,-\theta)}{\rho}
\end{aligned}
$$


and, from the fact that $S_{2}^{+,(2)}(\rho, \pi)=S_{2}^{-,(2)}(\rho,-\pi)=0$, we get the expression for the second Melnikov function

$$
\begin{aligned}
M_{2}(\rho)=r_{2}^{+}(\pi)-r_{2}^{-}(-\pi)= & \frac{F_{2}^{+,(2)}(\rho)+F_{2}^{-,(2)}(\rho)}{\rho} \pi \\
& +\frac{S_{2}^{+,(2)}(\rho, \pi)-S_{2}^{-,(2)}(\rho,-\pi)}{\rho} \\
= & \frac{F_{2}^{+,(2)}(\rho)+F_{2}^{-,(2)}(\rho)}{\rho} \pi .
\end{aligned}
$$

Now suppose that it vanishes identically, that is, $M_{2}(\rho) \equiv 0$. Then from Eq. (66) we have $F_{2}^{+,(2)}(\rho)=-F_{2}^{-,(2)}(\rho)$ and from Eqs. (64) the next relation holds:

$a_{2 j+1,2}^{-}=-a_{2 j+1,2}^{+}, \quad j=1, \ldots, n$.

From Eq. (64) it follows that $S_{2}^{+,(2)}(\rho, \theta)=-S_{2}^{-,(2)}(\rho, \theta)$. Also, using Eq. (65), we obtain

$$
\begin{aligned}
F_{2}^{+}(\rho) & =F_{2}^{+,(2)}(\rho)+F_{2}^{+,(1)}(\rho) \\
& =-\left(F_{2}^{-,(2)}(\rho)+F_{2}^{-,(2)}(\rho)\right)=-F_{2}^{-}(\rho),
\end{aligned}
$$

and similarly $S_{2}^{+}(\rho, \theta)=-S_{2}^{-}(\rho, \theta)$. This implies that

$S_{2}^{+}(\rho, \theta)-S_{2}^{-}(\rho,-\theta)=S_{2}^{+}(\rho, \theta)+S_{2}^{-}(\rho, \theta)=0$.

Now, from the form of $\hat{\alpha}_{2}^{ \pm}$and $\hat{\beta}_{2}^{ \pm}$given in (63), we have

$$
\begin{aligned}
\hat{\alpha}_{2}^{+}(\theta)-\hat{\alpha}_{2}^{-}(-\theta)= & \alpha_{2}^{+}(r, \theta)+h_{1}^{+}(r, \theta) \alpha_{1}^{+}(r, \theta) \\
& -\left(\alpha_{2}^{-}(r,-\theta)+h_{1}^{-}(r,-\theta) \alpha_{1}^{-}(r,-\theta)\right) \\
= & \alpha_{2}^{+}(r, \theta)+h_{1}^{+}(r, \theta) \alpha_{1}^{+}(r, \theta) \\
& -\left(\alpha_{2}^{+}(r, \theta)+h_{1}^{+}(r, \theta) \alpha_{1}^{+}(r, \theta)\right)=0 .
\end{aligned}
$$

Similarly we obtain $\hat{\beta}_{2}^{+}(\theta)+\hat{\beta}_{2}^{-}(-\theta)=0$ and, as $h_{2}^{ \pm}(r, \theta)=$ $\frac{1}{r}\left(\hat{\alpha}_{2}^{ \pm}(r, \theta)-\frac{\partial S_{2}^{ \pm}}{\partial r}\right)$, we get $h_{2}^{+}(r, \theta)-h_{2}^{-}(r,-\theta)=0$. Using all the above relations together we can write

$$
F_{2}^{+}(\rho)+F_{2}^{-}(\rho)=0, \quad S_{2}^{+}(\rho, \theta)-S_{2}^{-}(\rho,-\theta)=0,
$$

$h_{2}^{+}(\rho, \theta)-h_{2}^{-}(\rho,-\theta)=0, \quad \alpha_{2}^{+}(\rho, \theta)-\alpha_{2}^{-}(\rho,-\theta)=0$,

$\beta_{2}^{+}(\rho, \theta)+\beta_{2}^{-}(\rho,-\theta)=0, \quad r_{2}^{+}(\theta)-r_{2}^{-}(-\theta)=0$.

Now suppose that for $j=1, \ldots, m-1$ we have

$$
F_{j}^{+}(\rho)+F_{j}^{-}(\rho)=0, \quad S_{j}^{+}(\rho, \theta)-S_{j}^{-}(\rho,-\theta)=0,
$$

$h_{j}^{+}(\rho, \theta)-h_{j}^{-}(\rho,-\theta)=0, \quad \alpha_{j}^{+}(\rho, \theta)-\alpha_{j}^{-}(\rho,-\theta)=0$,

$$
\beta_{j}^{+}(\rho, \theta)+\beta_{j}^{-}(\rho,-\theta)=0, \quad r_{j}^{+}(\theta)-r_{j}^{-}(-\theta)=0 .
$$

Then the terms of order $\varepsilon^{m}$ of Eq. (8) in Proposition 2.3 can be written as

$$
\begin{aligned}
\rho r_{m}^{ \pm}(\theta)= & F_{m}^{ \pm}(\rho) \theta+S_{m}^{ \pm}(\rho, \theta)+\mathcal{O}_{\varepsilon^{m}} \\
& \times\left(-\frac{1}{2}\left(\rho+\varepsilon r_{1}^{ \pm}(\theta)+\cdots+\varepsilon^{m-1} r_{m-1}^{ \pm}(\theta)\right)^{2}\right) \\
& +\mathcal{O}_{\varepsilon^{m}}\left(\sum _ { i = 1 } ^ { m - 1 } \varepsilon ^ { i } \int _ { 0 } ^ { \theta } F _ { i } ^ { \pm } \left(\rho+\varepsilon r_{1}^{ \pm}(\psi)\right.\right. \\
& \left.\left.+\cdots+\varepsilon^{m-1} r_{m-1}^{ \pm}(\psi)\right) d \psi\right) \\
& +\mathcal{O}_{\varepsilon^{m}}\left(\sum_{i=1}^{m-1} S_{i}^{ \pm}\left(\rho+\varepsilon r_{1}^{ \pm}(\theta)+\cdots+\varepsilon^{m-1} r_{m-1}^{ \pm}(\theta)\right)\right)
\end{aligned}
$$

Now observe that as in the case $i=2$ under the hypothesis of induction (67) we have

$r_{m}^{+}(\theta)-r_{m}^{-}(-\theta)=\frac{F_{m}^{+}(\rho)+F_{m}^{-}(\rho)}{\rho} \theta+\frac{S_{m}^{+}(\rho, \theta)-S_{m}^{-}(\rho, \theta)}{\rho}$,

where the functions $F_{m}^{ \pm}, S_{m}^{ \pm}$and $h_{m}^{ \pm}$are obtained from the oneforms

$$
\begin{aligned}
-\Omega_{m}^{ \pm} & =-\sum_{j=1}^{m} \omega_{j}^{ \pm} h_{m-j}^{ \pm}=-\omega_{m}^{ \pm}-\sum_{j=1}^{m-1} \omega_{j}^{ \pm} h_{m-j}^{ \pm} \\
& =\hat{\alpha}_{m}^{ \pm}(r, \theta) d r+\hat{\beta}_{m}^{ \pm}(r, \theta) d \theta
\end{aligned}
$$

with

$$
\begin{aligned}
\hat{\alpha}_{m}^{ \pm}(r, \theta) & =\alpha_{m}^{ \pm}(r, \theta)+\sum_{j=1}^{m} h_{m-j}^{ \pm} \alpha_{j}^{ \pm}(r, \theta) \\
& =\alpha_{m}^{ \pm}(r, \theta)+\alpha_{m}^{ \pm,(m-1)}(r, \theta), \\
\hat{\beta}_{m}^{ \pm}(r, \theta) & =\beta_{m}^{ \pm}(r, \theta)+\sum_{j=1}^{m} h_{m-j}^{ \pm} \beta_{j}^{ \pm}(r, \theta) \\
& =\beta_{m}^{ \pm}(r, \theta)+\beta_{m}^{ \pm,(m-1)}(r, \theta) .
\end{aligned}
$$

Furthermore, it follows that

$$
\begin{aligned}
\alpha_{m}^{+,(m-1)}(r, \theta)-\alpha_{m}^{-,(m-1)}(r,-\theta)= & \sum_{j=1}^{m-1}\left(h_{m-j}^{+}(r, \theta) \alpha_{j}^{+}(r, \theta)\right. \\
& \left.-h_{m-j}^{-}(r,-\theta) \alpha_{j}^{-}(r,-\theta)\right) \\
= & \sum_{j=1}^{m-1}\left(h_{m-j}^{+}(r, \theta) \alpha_{j}^{+}(r, \theta)\right. \\
& \left.-h_{m-j}^{+}(r, \theta) \alpha_{j}^{+}(r, \theta)\right)=0 .
\end{aligned}
$$

Similarly, we obtain the vanishing relation $\beta_{m}^{+,(m-1)}(r, \theta)+$ $\beta_{m}^{-,(m-1)}(r,-\theta)=0$. Moreover, we can write

$$
\begin{aligned}
& F_{m}^{ \pm}(r)=F_{m}^{ \pm,(m)}(r)+F_{m}^{ \pm,(m-1)}(r), \quad \text { and } \\
& S_{m}^{ \pm}(r, \theta)=S_{m}^{ \pm,(m)}(r, \theta)+S_{m}^{ \pm,(m-1)}(r, \theta),
\end{aligned}
$$

with $F_{m}^{ \pm,(m)}, S_{m}^{ \pm,(m)}$ depending on the coefficients of order $\varepsilon^{m}$ of the one-forms (55) and they are given by

$$
\begin{aligned}
F_{m}^{ \pm,(m)}(r) & =-\frac{1}{2 \pi} \int_{0}^{2 \pi} \beta_{m}^{ \pm}(r, \theta) d \theta=\sum_{j=0}^{n} a_{2 j+1, m}^{ \pm} c_{2 j+2} r^{2 j+1}, \\
S_{m}^{ \pm,(m)}(r, \theta) & =\int_{0}^{\theta} \beta_{m}^{ \pm}(r, \psi) d \psi-F_{m}^{ \pm,(m)}(r) \theta \\
& =\sum_{j=0}^{n} a_{2 j+1, m}^{ \pm} \mathcal{C}_{2 j+2}(\theta) r^{2 j+2} .
\end{aligned}
$$

Here the functions $F_{m}^{ \pm,(m-1)}, S_{m}^{ \pm,(m-1)}$ depend on the coefficients of order $\varepsilon^{j}$ of the one-form (55), for every $j=1, \ldots, m-1$, and from the fact that $\beta_{m}^{+,(m-1)}(r, \theta)=-\beta_{m}^{-,{ }^{(m-1)}}(r, \theta)$ these functions satisfy

$$
\begin{gathered}
F_{m}^{+,(m-1)}(r)=-F_{m}^{-,(m-1)}(r), \quad \text { and } \\
S_{m}^{+,(m-1)}(r, \theta)=-S_{m}^{-,(m-1)}(r, \theta) .
\end{gathered}
$$

Now as

$S_{m}^{ \pm,(m-1)}(r, \theta)=\int_{0}^{\theta} \beta_{m}^{ \pm,(m-1)}(r, \psi) d \psi-F_{m}^{ \pm,(m-1)}(r) \theta$ 
we get, also using the symmetry of the functions $\beta$,

$$
\begin{aligned}
S_{m}^{+,(m-1)}(r, \theta)-S_{m}^{-,(m-1)}(r,-\theta)= & \int_{0}^{\theta} \beta_{m}^{+,(m-1)}(r, \psi) d \psi \\
& -\int_{0}^{-\theta} \beta_{m}^{-,(m-1)}(r, \psi) d \psi \\
= & \int_{0}^{\theta}\left(\beta_{m}^{+,(m-1)}(r, \psi)\right. \\
& \left.-\beta_{m}^{+,(m-1)}(r, \psi)\right) d \psi=0 .
\end{aligned}
$$

Then, from Eq. (68), we have

$$
\begin{aligned}
r_{m}^{+}(\theta)-r_{m}^{-}(-\theta)= & \frac{F_{m}^{+}(\rho)+F_{m}^{-}(\rho)}{\rho} \theta+\frac{S_{m}^{+}(\rho, \theta)-S_{m}^{-}(\rho,-\theta)}{\rho} \\
= & \frac{F_{m}^{+,(m)}(\rho)+F_{m}^{-,(m)}(\rho)}{\rho} \theta \\
& +\frac{S_{m}^{+,(m)}(\rho, \theta)-S_{m}^{-,(m)}(\rho,-\theta)}{\rho}
\end{aligned}
$$

and, from the fact that $S_{m}^{+,(m)}(\rho, \pi)=0=S_{m}^{-,(m)}(\rho,-\pi)$, we obtain

$$
\begin{aligned}
M_{m}=r_{m}^{+}(\pi)-r_{m}^{-}(-\pi)= & \frac{F_{m}^{+,(m)}(\rho)+F_{m}^{-,(m)}(\rho)}{\rho} \pi \\
& +\frac{S_{m}^{+,(m)}(\rho, \pi)-S_{m}^{-,(m)}(\rho,-\pi)}{\rho} \\
= & \frac{F_{m}^{+,(m)}(\rho)+F_{m}^{-,(m)}(\rho)}{\rho} \pi .
\end{aligned}
$$

This implies that, when $M_{m}(\rho) \equiv 0$, we have

$a_{2 j+1, m}^{-}=-a_{2 j+1, m}^{+}$, for $j=1, \ldots, m$.

From Eq. (70) it follows that $S_{m}^{+,{ }^{(m)}}(\rho, \theta)=-S_{m}^{-,{ }^{(m)}}(\rho, \theta)$. Also, from Eq. (71), we obtain

$$
\begin{aligned}
F_{m}^{+}(\rho) & =F_{m}^{+,(m)}(\rho)+F_{m}^{+,(m-1)}(\rho)=-\left(F_{m}^{-,(m)}(\rho)+F_{m}^{-,(m-1)}(\rho)\right) \\
& =-F_{m}^{-}(\rho),
\end{aligned}
$$

and similarly $S_{m}^{+}(\rho, \theta)=-S_{m}^{-}(\rho, \theta)$. This implies the next vanishing relation:

$S_{m}^{+}(\rho, \theta)-S_{m}^{-}(\rho,-\theta)=S_{m}^{+}(\rho, \theta)+S_{m}^{-}(\rho, \theta)=0$.

Consequently, from Eq. (68), the next difference also vanishes: $r_{m}^{+}(\theta)-r_{m}^{-}(-\theta)=0$.

Moreover, from the form of $\hat{\alpha}_{m}^{ \pm}$and $\hat{\beta}_{m}^{ \pm}$given by (69), we have

$$
\begin{aligned}
\hat{\alpha}_{m}^{+}(\theta)-\hat{\alpha}_{m}^{-}(-\theta)= & \alpha_{m}^{+}(r, \theta)+\sum_{j=1}^{m-1} h_{j}^{+}(r, \theta) \alpha_{j}^{+}(r, \theta) \\
& -\left(\alpha_{m}^{-}(r,-\theta)+\sum_{j=1}^{m-1} h_{j}^{-}(r,-\theta) \alpha_{j}^{-}(r,-\theta)\right) \\
= & \alpha_{m}^{+}(r, \theta)+\sum_{j=1}^{m-1} h_{j}^{+}(r, \theta) \alpha_{j}^{+}(r, \theta) \\
& -\left(\alpha_{m}^{+}(r, \theta)+\sum_{j=1}^{m-1} h_{j}^{+}(r, \theta) \alpha_{j}^{+}(r, \theta)\right)=0 .
\end{aligned}
$$

Similarly, we can obtain also that $\hat{\beta}_{m}^{+}(\theta)+\hat{\beta}_{m}^{-}(-\theta)=0$ and, as $h_{m}^{ \pm}(r, \theta)=\frac{1}{r}\left(\hat{\alpha}_{m}^{ \pm}-\frac{\partial S_{m}^{ \pm}}{\partial r}\right)$, we get $h_{m}^{+}(r, \theta)-h_{m}^{-}(r,-\theta)=0$.

In this way we obtain Eqs. (67) for $j=m$ and the result follows by induction.
Proof of Proposition 5.2. Using the tools described in Section 2 and writing $f_{i}^{ \pm}(y)=\sum_{j=0}^{n} a_{j, i}^{ \pm} y^{j}$, system (54) moves to its equivalent piecewise differential one-form

$$
\left\{\begin{array}{l}
d H+\sum_{i=0}^{N} \varepsilon^{i} \omega_{i}^{+}=0, \quad \text { if } \theta \in[0, \pi), \\
d H+\sum_{i=0}^{N} \varepsilon^{i} \omega_{i}^{-}=0, \quad \text { if } \theta \in[\pi, 2 \pi),
\end{array}\right.
$$

where $H=r^{2} / 2$ and $-\omega_{i}^{ \pm}=\alpha_{i}^{ \pm}(r, \theta) d r+\beta_{i}^{ \pm}(r, \theta) d \theta$, with

$$
\begin{gathered}
\alpha_{i}^{ \pm}(r, \theta)=\sum_{j=0}^{n} a_{j, i}^{ \pm} r^{j} \sin ^{j} \theta \cos \theta, \quad \text { and } \\
\beta_{i}^{ \pm}(r, \theta)=-\sum_{j=0}^{n} a_{j, i}^{ \pm} r^{j+1} \sin ^{j+1} \theta .
\end{gathered}
$$

Using these expressions we obtain

$$
\begin{aligned}
F_{1}^{ \pm}(r)= & -\sum_{j=0}^{[(n-1) / 2]} a_{2 j+1,1}^{ \pm} s_{2 j+2} r^{2 j+2}, \\
S_{1}^{ \pm}(r, \theta)=- & \sum_{j=0}^{[(n-1) / 2]} a_{2 j, 1}^{ \pm} \mathcal{S}_{2 j+1}(\theta) r^{2 j+1} \\
& -\sum_{j=0}^{[(n-1) / 2]} a_{2 j+1,1}^{ \pm} \mathcal{S}_{2 j+2}(\theta) r^{2 j+2} .
\end{aligned}
$$

Moreover, by Lemma 2.6, we can write also $\mathcal{S}_{2 j+2}(\theta)=$ $\sin \theta p_{2 j+1}(\cos \theta)$ and $\mathcal{S}_{2 j+1}(\theta)=\tilde{p}_{2 j+1}(\cos \theta)$ for some $p_{2 j+1}$ and $\tilde{p}_{2 j+1}$ polynomials of degree $2 j+1$ in $\cos \theta$.

Now, as

$\rho r_{1}^{ \pm}(\theta)=F_{1}^{ \pm}(\rho) \theta+S_{1}^{ \pm}(\rho, \theta)$,

it follows from this equation and the previous functions that the first Melnikov function, $M_{1}(\rho)=r_{1}^{+}(\pi)-r_{1}^{-}(-\pi)$, writes as

$$
\begin{aligned}
M_{1}(\rho)= & \frac{F_{1}^{+}(\rho)+F_{1}^{-}(\rho)}{\rho} \pi+\frac{S_{1}^{+}(\rho, \pi)-S_{1}^{-}(\rho,-\pi)}{\rho} \\
= & \sum_{j=0}^{[(n-1) / 2]}\left(a_{2 j, 1}^{-}-a_{2 j, 1}^{+}\right) \mathcal{S}_{2 j+1}(\pi) \rho^{2 j} \\
& -\sum_{j=0}^{[(n-1) / 2]}\left(a_{2 j+1,1}^{+}+a_{2 j+1,1}^{-}\right) s_{2 j+2} \rho^{2 j+1} .
\end{aligned}
$$

In this case, $M_{1}(\rho)$ is a polynomial of degree $n$ with monomials depending on the coefficients of the $\varepsilon$-perturbation of system (54). So (54) has at most $n$ limit cycles bifurcating from the origin up to a first order study. Moreover, as all the coefficients of $M_{1}(\rho)$ are linearly independent, we can chose a system such that the corresponding function $M_{1}$ has exactly $n$ simple zeros.

In order to study the second Melnikov function we suppose $M_{1}(\rho) \equiv 0$. In this case we get

$a_{2 j+1,1}^{+}=-a_{2 j+1,1}^{-}, \quad$ and $\quad a_{2 j, 1}^{+}=a_{2 j, 1}^{-}$.

Using (72), (73), and also the definition $h_{1}^{ \pm}(r, \theta)=\frac{1}{r}\left(\alpha_{1}^{ \pm}-\right.$ $\left.\frac{\partial S_{1}^{ \pm}}{\partial r}(r, \theta)\right)$, we can easily see that

$$
\begin{aligned}
F_{1}^{+}(\rho)+F_{1}^{-}(\rho)=0, & S_{1}^{+}(\rho, \theta)-S_{1}^{-}(\rho,-\theta)=0, \\
\alpha_{1}^{+}(\rho, \theta)-\alpha_{1}^{-}(\rho,-\theta)=0, & \beta_{1}^{+}(\rho, \theta)+\beta_{1}^{-}(\rho,-\theta)=0, \\
r_{1}^{+}(\theta)-r_{1}^{-}(-\theta)=0, & h_{1}^{+}(\rho, \theta)-h_{1}^{-}(\rho,-\theta)=0 .
\end{aligned}
$$


Taking into account the previous equalities and using the same induction procedure used for proving Theorem 1.3(b), we can show that $M_{k}(\rho)$ has the same form of $M_{1}(\rho)$ for every $k \in \mathbb{N}$. The proof of this result is quite similar to the proof of Theorem 1.3 and it is omitted.

\section{First order perturbations of Liénard families of low degree}

In this section we present two families for which the number of limit cycles increase with the order of perturbation but not as fast as the upper bound given in Theorem 1.1. As an example of the difficulties found during the implementation of this procedure we will present here only different families of lower degree. In the first one, the number of limit cycles with a second order study is bigger than the first order, but we have no more limit cycles with a third order or higher. For the second one, the number of limit cycles increases with the order, but also in this case this growth stops at a given order, fourth for this family. Typically, for a fixed family, a kind of saturation growth occurs. This phenomenon was also showed for the piecewise linear family in [11]. It is clear that which is this maximum number and for which order it is given are very difficult questions that involve too many computations in our approach. The first family has been studied up to order 6 and the second one only up to order 5 . We have not gone further because of the computational difficulties.

Proposition 6.1. For $n=1,2,3,4$, consider the polynomial system, in $\Sigma_{0}^{ \pm}$,

$X^{ \pm}:\left(x^{\prime}, y^{\prime}\right)=\left(-y+\sum_{i=1}^{N} \varepsilon^{i} f_{i, n}^{ \pm}(x), x\right)$,

where $f_{i, n}^{ \pm}$are real polynomials of degree $n$. Then $M_{1}$ has at most $[(n-1) / 2]$ simple zeros and $M_{N}$ has at most $n+[(n-1) / 2]$ simple zeros for $N=2, \ldots, 6$. Moreover there exist $f_{i, n}^{ \pm}$such that system (74), for $\varepsilon$ small enough, has exactly these number of limit cycles.

Proof. The proof is done in a case by case study. For each degree $n$, using the procedure described in Section 2, we obtain that $M_{N}(\rho)=P_{n, N}(\rho) / \rho^{d_{N}}$ where $P_{n, N}$ are polynomials of degree $m_{n, N}$ for $N=1,2, \ldots, 6$. For simplicity we have not indicated, in $P_{n, N}$, the dependence on the coefficients of the polynomials $f_{i, n}^{ \pm}$. Here the values of $d_{N}$ are $0,0,1,2,2,4$ for $N=1,2, \ldots, 6$ and the values of $m_{n, N}$ are given in the next table:

\begin{tabular}{|r|r|r|r|r|r|r|}
\hline$n$ & \multicolumn{1}{|l|}{$N$} & \multicolumn{1}{|c|}{$\mid$} \\
\hline & 1 & 2 & 3 & 4 & 5 & 6 \\
\hline 1 & 1 & 1 & 2 & 3 & 3 & 5 \\
\hline 2 & 1 & 2 & 4 & 6 & 7 & 10 \\
\hline 3 & 3 & 5 & 8 & 11 & 13 & 17 \\
\hline 4 & 3 & 6 & 10 & 14 & 17 & 22 \\
\hline
\end{tabular}

For the case corresponding to degree $n=2$, the first four polynomials $P_{2, N}$, taking $f_{i, 2}^{ \pm}(x)=a_{0, i}^{ \pm}+a_{1, i}^{ \pm} x+a_{2, i}^{ \pm} x^{2}$, are

$$
\begin{aligned}
P_{2,1}= & \frac{\pi}{2}\left(a_{11}^{+}+a_{11}^{-}\right) \rho, \\
P_{2,2}= & \frac{2}{9}\left(a_{11}^{+} a_{21}^{+}-a_{11}^{-} a_{21}^{-}\right) \rho^{2}-\frac{\pi}{8}\left(\pi\left(\left(a_{11}^{-}\right)^{2}-\left(a_{11}^{+}\right)^{2}\right)\right. \\
& \left.-4\left(a_{12}^{-}+a_{12}^{+}\right)\right) \rho+2\left(a_{01}^{+} a_{11}^{+}-a_{01}^{-} a_{11}^{-}\right), \\
P_{2,3}= & \frac{1}{24} \pi\left(a_{11}^{-}\left(a_{21}^{-}\right)^{2}+a_{11}^{+}\left(a_{21}^{+}\right)^{2}\right) \rho^{4}+\frac{1}{18}\left(3 \pi \left(\left(a_{11}^{+}\right)^{2} a_{21}^{+}\right.\right. \\
& \left.+\left(a_{11}^{-}\right)^{2} a_{21}^{-}\right)+4\left(a_{11}^{+} a_{22}^{+}-a_{11}^{-} a_{22}^{-}\right) \\
& \left.+4\left(a_{12}^{+} a_{21}^{+}-a_{12}^{-} a_{21}^{-}\right)\right) \rho^{3}+\frac{1}{48} \pi\left(\pi^{2}\left(\left(a_{11}^{-}\right)^{3}+\left(a_{11}^{+}\right)^{3}\right)\right.
\end{aligned}
$$

$$
\begin{aligned}
& -12 \pi\left(a_{11}^{-} a_{12}^{-}-a_{11}^{+} a_{12}^{+}\right) \\
& \left.+3\left(\left(a_{11}^{-}\right)^{3}+\left(a_{11}^{+}\right)^{3}\right)+24\left(a_{13}^{-}+a_{13}^{+}\right)\right) \rho^{2}+\frac{1}{2}\left(\pi \left(a_{01}^{+}\left(a_{11}^{+}\right)^{2}\right.\right. \\
& \left.+a_{01}^{-}\left(a_{11}^{-}\right)^{2}\right) \\
& \left.+4\left(a_{01}^{+} a_{12}^{+}+a_{02}^{+} a_{11}^{+}-a_{01}^{-} a_{12}^{-}-a_{02}^{-} a_{11}^{-}\right)\right) \rho+\frac{1}{2} \pi\left(\left(a_{01}^{-}\right)^{2} a_{11}^{-}\right. \\
& \left.+\left(a_{01}^{+}\right)^{2} a_{11}^{+}\right) \text {, } \\
& P_{2,4}=-\frac{206}{2025}\left(a_{11}^{-}\left(a_{21}^{-}\right)^{3}-a_{11}^{+}\left(a_{21}^{+}\right)^{3}\right) \rho^{6} \\
& -\frac{1}{648}\left(27\left(a_{11}^{-} a_{21}^{-}-a_{11}^{+} a_{21}^{+}\right)\left(a_{11}^{-} a_{21}^{-}+a_{11}^{+} a_{21}^{+}\right) \pi^{2}\right. \\
& -27\left(2\left(a_{11}^{-} a_{21}^{-} a_{22}^{-}+a_{11}^{+} a_{21}^{+} a_{22}^{+}\right)+a_{12}^{-}\left(a_{21}^{-}\right)^{2}+a_{12}^{+}\left(a_{21}^{+}\right)^{2}\right) \pi \\
& +32\left(a_{11}^{-} a_{21}^{-}-a_{11}^{+} a_{21}^{+}\right) \times \\
& \left.\left(a_{11}^{-} a_{21}^{-}+a_{11}^{+} a_{21}^{+}\right)\right) \rho^{5}-\frac{1}{648}\left(45\left(\left(a_{11}^{-}\right)^{3} a_{21}^{-}-\left(a_{11}^{+}\right)^{3} a_{21}^{+}\right) \pi^{2}\right. \\
& -108\left(\left(a_{11}^{-}\right)^{2} a_{22}^{-}+\left(a_{11}^{+}\right)^{2} a_{22}^{+}\right. \\
& \left.+2\left(a_{11}^{-} a_{12}^{-} a_{21}^{-}+a_{11}^{+} a_{12}^{+} a_{21}^{+}\right)\right) \pi+16\left(9 \left(a_{01}^{-} a_{11}^{-}\left(a_{21}^{-}\right)^{2}\right.\right. \\
& \left.-a_{01}^{+} a_{11}^{+}\left(a_{21}^{+}\right)^{2}\right)+9\left(a_{11}^{-} a_{23}^{-}-a_{11}^{+} a_{23}^{+}\right) \\
& +9\left(a_{12}^{-} a_{22}^{-}-a_{12}^{+} a_{22}^{+}\right)+9\left(a_{13}^{-} a_{21}^{-}-a_{13}^{+} a_{21}^{+}\right) \\
& \left.\left.+2\left(\left(a_{11}^{-}\right)^{3} a_{21}^{-}-\left(a_{11}^{+}\right)^{3} a_{21}^{+}\right)\right)\right) \rho^{4} \\
& -\frac{1}{1152}\left(3\left(a_{11}^{-}-a_{11}^{+}\right)\left(a_{11}^{-}+a_{11}^{+}\right)\left(\left(a_{11}^{-}\right)^{2}+\left(a_{11}^{+}\right)^{2}\right) \pi^{4}\right. \\
& -72\left(\left(a_{11}^{-}\right)^{2} a_{12}^{-}+\left(a_{11}^{+}\right)^{2} a_{12}^{+}\right) \pi^{3} \\
& +36\left(\left(a_{11}^{-}\right)^{4}-\left(a_{11}^{+}\right)^{4}+8\left(a_{11}^{-} a_{13}^{-}-a_{11}^{+} a_{13}^{+}\right)\right. \\
& \left.+4\left(\left(a_{12}^{-}\right)^{2}-\left(a_{12}^{+}\right)^{2}\right)\right) \pi^{2}-72\left(3 \left(\left(a_{11}^{-}\right)^{2} a_{12}^{-}\right.\right. \\
& \left.\left.+\left(a_{11}^{+}\right)^{2} a_{12}^{+}\right)+8\left(a_{14}^{-}+a_{14}^{+}\right)\right) \pi+512\left(a_{01}^{-}\left(a_{11}^{-}\right)^{2} a_{21}^{-}\right. \\
& \left.\left.-a_{01}^{+}\left(a_{11}^{+}\right)^{2} a_{21}^{+}\right)\right) \rho^{3} \\
& -\frac{1}{24}\left(-3\left(a_{01}^{-}\left(a_{11}^{-}\right)^{3}-a_{01}^{+}\left(a_{11}^{+}\right)^{3}\right) \pi^{2}\right. \\
& +12\left(2\left(a_{01}^{-} a_{11}^{-} a_{12}^{-}+a_{01}^{+} a_{11}^{+} a_{12}^{+}\right)+a_{02}^{-}\left(a_{11}^{-}\right)^{2}\right. \\
& \left.+a_{02}^{+}\left(a_{11}^{+}\right)^{2}\right) \pi+16\left(\left(a_{01}^{-}\right)^{2} a_{11}^{-} a_{21}^{-}-\left(a_{01}^{+}\right)^{2} a_{11}^{+} a_{21}^{+}\right. \\
& +3\left(a_{01}^{+} a_{13}^{+}-a_{01}^{-} a_{13}^{-}+a_{02}^{+} a_{12}^{+}-a_{02}^{-} a_{12}^{-}\right. \\
& \left.\left.\left.+a_{03}^{+} a_{11}^{+}-a_{03}^{-} a_{11}^{-}\right)\right)\right) \rho^{2}-\frac{\pi}{2}\left(\left(a_{01}^{-}\right)^{2} a_{12}^{-}+\left(a_{01}^{+}\right)^{2} a_{12}^{+}\right. \\
& \left.+2\left(a_{01}^{-} a_{02}^{-} a_{11}^{-}+a_{01}^{+} a_{02}^{+} a_{11}^{+}\right)\right) \rho \\
& -\frac{2}{3}\left(\left(a_{01}^{-}\right)^{3} a_{11}^{-}-\left(a_{01}^{+}\right)^{3} a_{11}^{+}\right) \text {. }
\end{aligned}
$$

We have written the parameters $a_{i, j}$ as $a_{i j}$ so write in short. Moreover, the polynomials $P_{2,5}$ and $P_{2,6}$ are not written here because of the size of them. Instead of $P_{2,4}$ has 62 monomials, $P_{2,5}$ and $P_{2,6}$ have 152 and 350 , respectively.

Clearly, $P_{2,1}$ has no positive zeros, consequently there are no limit cycles bifurcating from the period annulus up to order 1 . Let us continue assuming $M_{1}(\rho) \equiv 0$. Under this assumption, $P_{2,2}$ is a polynomial of degree 2 with arbitrary coefficients. Then there exists a choice of perturbed coefficients such that $P_{2,2}$, and also $M_{2}$, has exactly 2 simple zeros and from the period annulus we can get 2 limit cycles for system (74), for $\varepsilon$ small enough. When $M_{1}(\rho) \equiv M_{2}(\rho) \equiv 0$, then all the coefficients of $M_{3}$ of degree greater than 2 vanish. The same simplification occurs for $M_{4}, M_{5}$, and $M_{6}$ when the previous $M_{N}$ vanish identically. Consequently, for $\varepsilon$ small enough, no more than 2 limit cycles can bifurcate from the period annulus up to orders $3,4,5$, and 6 . It can be easily checked that for system

$$
\begin{aligned}
& X^{+}:\left(x^{\prime}, y^{\prime}\right)=\left(-y+\left(\frac{9}{2} x^{2}+x+\frac{e_{0}}{2}\right) \varepsilon+2 e_{1} x \varepsilon^{2}, x\right), \\
& X^{-}:\left(x^{\prime}, y^{\prime}\right)=(-y-x \varepsilon, x),
\end{aligned}
$$


defined in $\Sigma_{0}^{ \pm}$, we have $M_{1}(\rho) \equiv 0$ and $M_{2}(\rho)=\rho^{2}+\pi e_{1} \rho$ $+e_{0}$.

Using the same simplification procedure, the degrees of the polynomials $P_{n, N}$ decrease. Next table shows which they are after the simplifications:

\begin{tabular}{|l|l|l|l|l|l|l|}
\hline$n$ & \multicolumn{1}{|c|}{$N$} & \multicolumn{5}{|c|}{} \\
\hline & 1 & 2 & 3 & 4 & 5 & 6 \\
\hline 1 & 1 & 1 & 1 & 1 & 1 & 1 \\
\hline 2 & 1 & 2 & 2 & 2 & 2 & 2 \\
\hline 3 & 3 & 4 & 4 & 4 & 4 & 4 \\
\hline 4 & 3 & 6 & 6 & 6 & 6 & 6 \\
\hline
\end{tabular}

We remark that the above table does not give the number of limit cycles, only the degrees of $P_{n, N}$ after the simplification procedure. The number of limit cycles given in the statement follows studying the independence of the parameters of $P_{n, N}$ for each degree $n$ and order $N$. We remark that although $P_{4, N}$ has degree 6 , the coefficient of degree 5 vanishes, consequently $M_{2}$ can have only 5 positive simple zeros.

Finally, we show explicit systems of degrees 1, 3 and 4 such that the corresponding functions $M_{1}$ are identically zero and the functions $M_{2}$ have 1,4 and 5 positive simple zeros, respectively.

Using the described procedure we can prove that the linear system

$$
X^{+}:\left(x^{\prime}, y^{\prime}\right)=\left(-y+\varepsilon\left(e_{0} x+1\right)+\varepsilon^{2} x, x\right) \text {, }
$$$$
X^{-}:\left(x^{\prime}, y^{\prime}\right)=\left(-y-\varepsilon e_{0} x, x\right) \text {, }
$$

defined in $\Sigma_{0}^{ \pm}$, has $M_{1}(\rho) \equiv 0$ and $M_{2}(\rho)=\pi \rho / 2+2 e_{0}$. Similarly, the cubic system

$$
\begin{gathered}
X^{+}:\left(x^{\prime}, y^{\prime}\right)=\left(-y+\left(\frac{1}{63 e_{0}} x^{3}+\frac{135}{2} e_{0} x^{2}+x-\frac{945}{2} e_{0}^{2}\right.\right. \\
\left.\left.+\frac{63}{2} e_{2} e_{0}\right) \varepsilon+2 e_{1} x \varepsilon^{2}, x\right), \\
X^{-}:\left(x^{\prime}, y^{\prime}\right)=\left(-y-\varepsilon\left(\frac{1}{63 e_{0}} x^{3}+x\right)+\frac{8}{3} e_{3} x^{3} \varepsilon^{2}, x\right),
\end{gathered}
$$

defined in $\Sigma_{0}^{ \pm}$, has $M_{1}(\rho) \equiv 0$ and $M_{2}(\rho)=\rho^{4}+\pi e_{3} \rho^{3}+e_{2} \rho^{2}+$ $\pi e_{1} \rho-63 e_{0}\left(15 e_{0}-2 e_{2}\right)$. We remark that there are values of $e_{i}$, $i=0, \ldots, 3$ such that $M_{2}$ has 4 positive simple zeros. For example for $e_{0}=1, e_{1}=-417 /(31 \pi), e_{2}=467 / 31, e_{3}=-207 /(31 \pi)$ we have $M_{4}(\rho)=(\rho-1)(\rho-2)(\rho-3)(\rho-21 / 31)$.

Finally, the quartic system

$$
\begin{aligned}
& X^{+}:\left(x^{\prime}, y^{\prime}\right)=\left(-y+\left(\frac{525}{58} x^{4}+x^{3}+e_{0} x\right) \varepsilon\right. \\
& \left.+\left(\frac{8}{3} e_{3} x^{3}+2 e_{1} x\right) \varepsilon^{2}, x\right), \\
& X^{-}:\left(x^{\prime}, y^{\prime}\right)=\left(-y+\left(-x^{3}+\left(\frac{555}{58} e_{0}+\frac{15}{14} e_{4}\right) x^{2}-e_{0} x\right.\right. \\
& \left.\left.-\frac{185}{174} e_{0}^{2}-\frac{5}{42} e_{0} e_{4}+\frac{1}{2} e_{2}\right) \varepsilon, x\right),
\end{aligned}
$$

defined in $\Sigma_{0}^{ \pm}$, has $M_{1}(\rho) \equiv 0$ and

$$
\begin{aligned}
M_{2}(\rho)= & \rho^{6}+e_{4} \rho^{4}+\pi e_{3} \rho^{3}+e_{2} \rho^{2}+\pi e_{1} \rho \\
& -e_{0}\left(1295 e_{0}^{2}+145 e_{0} e_{4}-609 e_{2}\right) / 609
\end{aligned}
$$

In general the above polynomial can have at most 5 positive zeros. Moreover, there exist values of $e_{0}, \ldots, e_{4}$ such that it has 5 positive simple zeros. For example, when $e_{0}=3 / 2, e_{1}=$ $-830235 /(841 \pi), e_{2}=644273 / 1682, e_{3}=45885 /(841 \pi), e_{4}=$ $-68047 / 1682$, we have that $M_{2}$ has five positive simple zeros at $1,2,3,4,-5+\sqrt{1534} / 58$ and one negative at $-5-\sqrt{1534} / 58$.

Consequently, there exist values of the perturbation parameters such that system (74), for $\varepsilon$ small enough and $n=1,2,3,4$, has 1, 2, 4, 5 limit cycles.
Proposition 6.2. For $n=1,2,3,4$, consider the polynomial system

$$
\begin{aligned}
X^{ \pm}:\left(x^{\prime}, y^{\prime}\right) & =\left(-y+f^{ \pm}(x, \varepsilon), x+g^{ \pm}(x, \varepsilon)\right) \\
& =\left(-y+\sum_{i=1}^{N} \varepsilon^{i} f_{i, n}^{ \pm}(x), x+\sum_{i=1}^{N} \varepsilon^{i} g_{i, n}^{ \pm}(x)\right),
\end{aligned}
$$

defined in $\Sigma_{0}^{ \pm}$, where $f_{i, n}^{ \pm}$and $g_{i, n}^{ \pm}$are polynomials of degree $n$. Then the functions $M_{N}$ have at most $n, 2 n-1,2 n$, 3n, 3n simple zeros for $N=1,2, \ldots, 5$. Moreover there exist $f_{i, n}^{ \pm}$and $g_{i, n}^{ \pm}$such that system (75), for $\varepsilon$ small enough, has these number of limit cycles.

Proof. The proof follows the same procedure as we have described in the proof of Proposition 6.1. In fact the Melnikov functions take the same form, $M_{N}(\rho)=P_{n, N}(\rho) / \rho^{d_{N}}$, but with different degrees and coefficients. In this case the values of $d_{N}$ are $0,0,1,2,3$ for $N=1,2, \ldots, 5$ and the values of the degrees of the polynomials $P_{n, N}$ are $m_{n, 1}=n$ and $m_{n, N}=N n-1$ for $N=2, \ldots, 5$.

We note that the maximum number of limit cycles appears up to $\operatorname{order} N=4$. Now we will only provide explicit examples exhibiting this maximum number of limit cycles, for each degree $n$ in the statement. The studies of lower order can be done similarly.

The linear Liénard system (75) with $N=4$ given by

$$
\begin{aligned}
& X^{+}:\left(x^{\prime}, y^{\prime}\right)=\left(-y+\left(e_{0}+x\right) \varepsilon+2 x \varepsilon^{4}, x-\frac{e_{1}}{2 e_{0}} \varepsilon\right), \\
& X^{-}:\left(x^{\prime}, y^{\prime}\right)=\left(-y+\left(e_{0}-x\right) \varepsilon+\frac{1}{2}\left(-4 e_{0}+e_{2}\right) \varepsilon^{3},\right. \\
& \left.x-\frac{e_{1}}{2 e_{0}} \varepsilon+2 e_{0} \varepsilon^{2}\right),
\end{aligned}
$$

has $M_{1}(\rho) \equiv M_{2}(\rho) \equiv M_{3}(\rho) \equiv 0$ and

$$
M_{4}(\rho)=\frac{1}{\rho^{2}}\left(\pi \rho^{3}+e_{2} \rho^{2}+\pi e_{1} \rho+\frac{4}{3} e_{0}^{3}\right) \text {. }
$$

The quadratic system (75) with $N=4$ and

$$
\begin{aligned}
& f^{+}(x, \varepsilon)=\left(x^{2}+x+e_{0}\right) \varepsilon+\frac{81 e_{4}+180 e_{0}+4}{18} x^{2} \varepsilon^{3}, \\
& g^{+}(x, \varepsilon)= x^{2} \varepsilon^{2}+6 e_{5} x^{2} \varepsilon^{3}+\frac{9 e_{2}-4 e_{0}^{2}-36 e_{0}}{18} \varepsilon^{4}, \\
& f^{-}(x, \varepsilon)= \frac{1}{3}\left(-5 x^{2}-3 x+3 e_{0}\right) \varepsilon-\frac{e_{1}}{e_{0}} \varepsilon^{2}-\frac{20 e_{0}}{3} x \varepsilon^{3} \\
&+\frac{2\left(3 e_{0} e_{3}+5 e_{1}\right)}{3 e_{0}} x \varepsilon^{4}, \\
& g^{-}(x, \varepsilon)= \frac{1}{9}\left(7 x^{2}+18 e_{0}\right) \varepsilon^{2}+\frac{1}{e_{0}}\left(6 e_{0} e_{5} x^{2}-e_{1}\right) \varepsilon^{3}, \\
& \text { has } M_{1}(\rho) \equiv M_{2}(\rho) \equiv M_{3}(\rho) \equiv 0 \text { and } \\
& M_{4}(\rho)=\frac{1}{\rho^{2}}\left(\frac{19292}{54675} \rho^{6}+\pi e_{5} \rho^{5}+e_{4} \rho^{4}+\pi e_{3} \rho^{3}\right. \\
&\left.+e_{2} \rho^{2}+\pi e_{1} \rho+\frac{4}{3} e_{0}^{3}\right) .
\end{aligned}
$$

The cubic system (75) with $N=4$ and

$$
\begin{aligned}
& X^{+}:\left\{\begin{aligned}
x^{\prime}= & -y+\left(a_{8} x^{2}+x+a_{0}\right) \varepsilon+\left(\frac{53}{120} x^{3}+a_{7} x\right) \varepsilon^{2}, \\
y^{\prime}= & x+x^{2} \varepsilon^{3},
\end{aligned}\right. \\
& X^{-}:\left\{\begin{aligned}
x^{\prime}= & -y+\left(-\frac{571}{1525} a_{8} x^{2}-x+a_{0}\right) \varepsilon+A_{1}(x) \varepsilon^{2}+A_{2}(x) \varepsilon^{3} \\
& +a_{5} x^{3} \varepsilon^{4}, \\
y^{\prime}= & x+x^{3} \varepsilon+\left(\frac{318}{1525} a_{8} x^{2}+2 a_{0}\right) \varepsilon^{2}+A_{3}(x) \varepsilon^{3}+a_{4} x^{2} \varepsilon^{4},
\end{aligned}\right.
\end{aligned}
$$


where

$A_{1}(x)=-\frac{163}{120} x^{3}+a_{6} x^{2}-a_{7} x+a_{1}$,

$A_{2}(x)=\left(-\frac{343228}{2325625} a_{8}^{2}-\frac{11}{12} a_{7}\right) x^{3}-\frac{2284}{1525} a_{0} a_{8} x+a_{2}$,

$A_{3}(x)=\left(\frac{318}{1525} a_{7} a_{8}+\frac{42}{5} a_{0}+\frac{1}{3} a_{6}+1\right) x^{2}+a_{3} x+2 a_{0} a_{7}+a_{1}$,

has $M_{1}(\rho) \equiv M_{2}(\rho) \equiv M_{3}(\rho) \equiv 0$. And choosing

$a_{0}=e_{0}$,

$a_{1}=-\frac{4}{3} \frac{e_{1}}{a_{0}}$,

$a_{2}=\frac{2}{3} e_{2}+\frac{318}{1525} a_{0}^{2} a_{8}-a_{1} a_{7}-2 a_{0}$,

$a_{3}=\frac{16}{3} e_{3}-\frac{4568}{1525} a_{0} a_{7} a_{8}+8 a_{6} a_{0}-\frac{2284}{1525} a_{1} a_{8}$,

$a_{4}=-2 e_{4}+\frac{2}{3} a_{0} a_{8}^{2}+\frac{23}{4} a_{0} a_{7}+\frac{1}{3} a_{7} a_{6}+\frac{163}{40} a_{1}+\frac{106}{1525} a_{8}$,

$a_{5}=\frac{32}{9} e_{5}-\frac{343228}{2325625} a_{7} a_{8}^{2}-\frac{64316}{22875} a_{0} a_{8}-\frac{376}{13725} a_{8} a_{6}$

$$
+\frac{636}{1525} a_{8}+\frac{1}{72}
$$

$a_{6}=\frac{48}{61} e_{6}-\frac{24}{61}+\frac{353934}{2325625} a_{7} a_{8}-\frac{201099590064}{5408531640625} a_{8}^{3}-\frac{159}{305} a_{0}$,

$a_{7}=-\frac{2048}{159} e_{7}+\frac{3439367744}{5546615625} a_{8}^{2}$,

$a_{8}=\frac{448350000}{150098827} e_{8}$,

we obtain

$$
\begin{aligned}
M_{4}(\rho)= & \frac{1}{\rho^{2}}\left(\frac{13863}{655360} \pi \rho^{9}+e_{8} \rho^{8}+\pi e_{7} \rho^{7}+e_{6} \rho^{6}+\pi e_{5} \rho^{5}\right. \\
& \left.+e_{4} \rho^{4}+\pi e_{3} \rho^{3}+e_{2} \rho^{2}+\pi e_{1} \rho+e_{0}^{3}\right) .
\end{aligned}
$$

The quartic system (75) with $N=4$ and

$X^{+}:\left\{\begin{aligned} x^{\prime}= & -y+\left(a_{10} x^{2}+a_{0}+x\right) \varepsilon \\ & +\left(a_{9} x^{4}+\frac{4021}{348} a_{11} x^{3}+a_{1}\right) \varepsilon^{2}+a_{6} x^{4} \varepsilon^{3}+a_{3} x \varepsilon^{4}, \\ y^{\prime}= & x+A_{1}(x) \varepsilon-\frac{617}{58} a_{11} x^{3} \varepsilon^{2}+A_{2}(x) \varepsilon^{3}+\varepsilon^{4} a_{5} x^{3},\end{aligned}\right.$
$X^{-}:\left\{\begin{aligned} x^{\prime}= & -y+A_{3}(x) \varepsilon+-\frac{617}{58} a_{11} x^{3} \varepsilon^{2}+A_{4}(x) \varepsilon^{3}+a_{5} x^{3} \varepsilon^{4}, \\ y^{\prime}= & x+A_{5}(x) \varepsilon^{2}+A_{6}(x) \varepsilon^{3},\end{aligned}\right.$

with

$$
\begin{aligned}
A_{1}(x)= & x^{4}+\left(\frac{11177145}{373984} a_{11}^{2}+\frac{2551}{403} a_{10}\right) x^{2}-x+a_{0}, \\
A_{2}(x)= & \left(\frac{41642856783675}{139864032256} a_{11}^{4}+\frac{1250598335}{9419722} a_{10} a_{11}^{2}\right. \\
& \left.+\frac{21416500}{1461681} a_{10}^{2}+\frac{16}{3} a_{0}-\frac{11}{12} a_{8}\right) x^{3} \\
& +\left(\frac{11177145}{93496} a_{0} a_{11}^{2}+\frac{10204}{403} a_{0} a_{10}\right) x, \\
A_{3}(x)= & x^{4}+\left(\frac{11177145}{373984} a_{11}^{2}+\frac{2551}{403} a_{10}\right) x^{2}-x+a_{0}, \\
A_{4}(x)= & \left(\frac{41642856783675}{139864032256} a_{11}^{4}+\frac{1250598335}{9419722} a_{10} a_{11}^{2}\right. \\
& \left.+\frac{21416500}{1461681} a_{10}^{2}+\frac{16}{3} a_{0}-\frac{11}{12} a_{8}\right) x^{3} \\
& +\frac{1}{93496} a_{0}\left(11177145 a_{11}^{2}+2367328 a_{10}\right) x,
\end{aligned}
$$

$$
\begin{aligned}
A_{5}(x)= & \frac{33}{70} x^{4}+a_{8} x^{3}+\left(\frac{3725715}{373984} a_{11}^{2}+\frac{2954}{1209} a_{10}\right) x^{2}+2 a_{0}, \\
A_{6}(x)= & \left(\frac{16091363085}{21691072} a_{11}^{3}+\frac{129441493}{701220} a_{10} a_{11}-\frac{37}{15} a_{9}\right) x^{4} \\
& +\left(\frac{7375}{116} a_{0} a_{11}+a_{7}\right) x^{2}+a_{1},
\end{aligned}
$$

has $M_{1}(\rho) \equiv M_{2}(\rho) \equiv M_{3}(\rho) \equiv 0$. Choosing the values

$a_{0}=e_{0}$

$a_{1}=\frac{e_{1}}{e_{0}}$

$a_{2}=\frac{1}{2} e_{2}-2 a_{0}+\frac{2954}{1209} a_{0}^{2} a_{10}+\frac{3725715}{373984} a_{0}^{2} a_{11}^{2}$,

$a_{3}=2 e_{3}+\frac{11177145}{186992} a_{1} a_{11}^{2}+\frac{5102}{403} a_{1} a_{10}$,

$a_{4}=\frac{3}{2} e_{4}-\frac{2954}{3627} a_{10}+\frac{1}{5} a_{0}^{2}-\frac{1241905}{373984} a_{11}^{2}-\frac{2}{3} a_{0} a_{10}^{2}$

$-\frac{3673}{116} a_{1} a_{11}-\frac{23}{4} a_{0} a_{8}$,

$a_{5}=\frac{8}{3} e_{5}+\frac{41269745055}{21691072} a_{11}^{3} a_{0}+\frac{916501}{2418} a_{10} a_{0} a_{11}$

$+\frac{3725715}{186992} a_{11}^{2} a_{7}+\frac{8}{3} a_{0} a_{9}$

$+\frac{5908}{1209} a_{10} a_{7}+4 a_{1}-\frac{1}{72} a_{11}$,

$a_{6}=\frac{75}{74} e_{6}+\frac{277}{111}-\frac{957035384280675}{28182602499584} a_{10} a_{11}^{4}$

$-\frac{246061222155}{60738367456} a_{10}^{2} a_{11}^{2}$

$+\frac{58101015}{1495936} a_{11}^{2} a_{8}+\frac{40871}{4836} a_{10} a_{8}+\frac{17041717574}{65385376173} a_{10}^{3}$

$-\frac{25}{37} a_{11} a_{7}$

$-\frac{1397742487944051375}{26153455119613952} a_{11}^{6}-\frac{56001305}{13837408} a_{0} a_{11}^{2}$

$+\frac{686648}{313131} a_{0} a_{10}$

$a_{7}=-\frac{12}{5} e_{7}-\frac{416237010695545095}{32448455483392} a_{11}^{5}$

$-\frac{993055562624097}{174830040320} a_{11}^{3} a_{10}+\frac{82710873}{3739840} a_{11}^{2} a_{9}$

$-\frac{1751870985349}{2825916600} a_{10}^{2} a_{11}-\frac{7375}{116} a_{0} a_{11}+\frac{242861}{60450} a_{10} a_{9}$

$+\frac{5553}{928} a_{11} a_{8}$

$a_{8}=\frac{22050}{43123} e_{8}+\frac{408588973833}{812413343612} a_{10} a_{11}^{2}-\frac{64879}{43123} a_{9} a_{11}$

$-\frac{1372}{43123} a_{0}$

$+\frac{14949785585339325}{1507839165743872} a_{11}^{4}-\frac{12575899204}{21010689921} a_{10}^{2}$,

$a_{9}=-\frac{320}{33} e_{9}-\frac{1724620185}{834272} a_{11}^{3}-\frac{2467922575}{4984056} a_{10} a_{11}$,

$a_{10}=\frac{1799445375}{1023106108} e_{10}-\frac{1075494673575}{237360617056} a_{11}^{2}$,

$a_{11}=-\frac{556800}{211631} e_{11}$,

we have that

$\begin{aligned} M_{4}(\rho)=\frac{1}{\rho^{2}}( & \frac{328474}{6670125} \rho^{12}+\pi e_{11} \rho^{11}+e_{10} \rho^{10}+\pi e_{9} \rho^{9} \\ & +e_{8} \rho^{8}+\pi e_{7} \rho^{7}\end{aligned}$ 


$$
\begin{aligned}
& +e_{6} \rho^{6}+\pi e_{5} \rho^{5}+e_{4} \rho^{4}+\pi e_{3} \rho^{3}+e_{2} \rho^{2} \\
& \left.+\pi e_{1} \rho+\frac{4}{3} e_{0}^{3}\right)
\end{aligned}
$$

For all the studied cases, $n=1,2,3,4$, the first three Melnikov functions vanish identically and the fourth, $M_{4}$, can be written with arbitrary coefficients, $e_{j}$. Consequently up to a study of fourth order and for $\varepsilon$ small enough, system (75) has $3 n$ limit cycles, bifurcating from some chosen $3 n$ circumferences, for $n=1,2,3,4$.

\section{Acknowledgments}

This work has been realized thanks to the MINECO grants MTM2013-40998-P and MTM2016-77278-P (FEDER), the AGAUR grant 2014 SGR568, the European Community grants FP7-PEOPLE2012-IRSES 316338 and 318999, and the Brazilian FAPESP grants 2012/18780-0, 2013/24541-0 and 2017/03352-6.

\section{References}

[1] M. di Bernardo, C.J. Budd, A.R. Champneys, P. Kowalczyk, Piecewise-Smooth Dynamical Systems, in: Applied Mathematical Sciences, vol. 163, SpringerVerlag London, Ltd., London, 2008. Theory and applications.

[2] A.F. Filippov, Differential Equations with Discontinuous Righthand Sides, in: Mathematics and its Applications (Soviet Series), vol. 18, Kluwer Academic Publishers Group, Dordrecht, 1988.

[3] A.A. Andronov, A.A. Vitt, S.E. Khaǐkin, Theory of Oscillators, Dover Publications, Inc., New York, 1987. Translated from the Russian by F. Immirzi, Reprint of the 1966 translation.

[4] I.D. Iliev, The number of limit cycles due to polynomial perturbations of the harmonic oscillator, Math. Proc. Cambridge Philos. Soc. 127 (2) (1999) 317322.

[5] A. Buică, On the equivalence of the Melnikov functions method and the averaging method, Qual. Theory Dyn. Syst. 16 (3) (2017) 547-560.

[6] M. Han, V.G. Romanovski, X. Zhang, Equivalence of the Melnikov function method and the averaging method, Qual. Theory Dyn. Syst. 15 (2) (2016) 471 479.

[7] A. Buică, J. Giné, J. Llibre, Bifurcation of limit cycles from a polynomial degenerate center, Adv. Nonlinear Stud. 10 (3) (2010) 597-609.

[8] A. Gasull, J. Torregrosa, A relation between small amplitude and big limit cycles, Rocky Mountain J. Math. 31 (4) (2001) 1277-1303.

[9] E. Freire, E. Ponce, F. Rodrigo, F. Torres, Bifurcation sets of continuous piecewise linear systems with two zones, Internat. J. Bifur. Chaos Appl. Sci. Engrg. 28 (11) (1998) 2073-2097.
[10] J.C. Medrado, J. Torregrosa, Uniqueness of limit cycles for sewing planar piecewise linear systems, J. Math. Anal. Appl. 431 (1) (2015) 529-544.

[11] C. Buzzi, C. Pessoa, J. Torregrosa, Piecewise linear perturbations of a linear center, Discrete Contin. Dyn. Syst. 33 (9) (2013) 3915-3936.

[12] Y. Tang, J. Llibre, Limit cycles of discontinuous piecewise quadratic and cubic polynomial perturbations of a linear center, Preprint, 2016.

[13] J. Llibre, A.C. Mereu, Limit cycles for discontinuous generalized Lienard polynomial differential equations, Electron. J. Differential Equations 195 (8) (2013).

[14] A. Lins, W. de Melo, C.C. Pugh, On Liénard's equation, in: Geometry and Topology (Proc. III Latin Amer. School of Math., Inst. Mat. Pura Aplicada CNPq, Rio de Janeiro, 1976), in: Lecture Notes in Math., vol. 597, Springer, Berlin, 1977, pp. 335-357.

[15] C. Zuppa, Order of cyclicity of the singular point of Liénard's polynomial vector fields, Bol. Soc. Brasil. Mat. 12 (2) (1981) 105-111.

[16] C. Li, J. Llibre, Uniqueness of limit cycles for Liénard differential equations of degree four, J. Differential Equations 252 (4) (2012) 3142-3162.

[17] P. De Maesschalck, F. Dumortier, Classical Liénard equations of degree $n \geq 6$ can have $\left[\frac{n-1}{2}\right]+2$ limit cycles, J. Differential Equations 250 (4) (2011) 21622176.

[18] F. Dumortier, D. Panazzolo, R. Roussarie, More limit cycles than expected in Liénard equations, Proc. Amer. Math. Soc. 135 (6) (2007) 1895-1904.

[19] B. Coll, A. Gasull, R. Prohens, First Lyapunov constants for non-smooth Liénard differential equations, in: Proceedings of the 2nd Catalan Days on Applied Mathematics, (Odeillo, 1995), in: Collect. Études, Presses Univ. Perpignan, Perpignan, 1995, pp. 77-83.

[20] G.A. Leonov, Limit cycles of the Lienard equation with discontinuous coefficients, Dokl. Akad. Nauk 426 (1) (2009) 47-50.

[21] L. Sheng, Limit cycles of a class of piecewise smooth Liénard systems, Internat. J. Bifur. Chaos Appl. Sci. Engrg. 26 (1) (2016) 165000910.

[22] P.T. Cardin, J. Torregrosa, Limit cycles in planar piecewise linear differential systems with nonregular separation line, Physica D 337 (2016) 67-82.

[23] A. Gasull, J. Torregrosa, Center-focus problem for discontinuous planar differential equations, Internat. J. Bifur. Chaos Appl. Sci. Engrg. 13 (7) (2003) 17551765. Dynamical systems and functional equations (Murcia, 2000).

[24] J.-P. Françoise, Successive derivatives of a first return map, application to the study of quadratic vector fields, Ergodic Theory Dynam. Systems 16 (1) (1996) 87-96.

[25] J.-P. Françoise, The first derivative of the period function of a plane vector field, in: Proceedings of the Symposium on Planar Vector Fields, Lleida, 1996, vol. 41, 1997, pp. 127-134.

[26] J.-P. Françoise, The successive derivatives of the period function of a plane vector field, J. Differential Equations 146 (2) (1998) 320-335.

[27] M. Abramowitz, I.A. Stegun, HandBook of Mathematical Functions with Formulas, Graphs, and Mathematical Tables, in: National Bureau of Standards Applied Mathematics Series, vol. 55, U.S. Government Printing Office, Washington D.C., 1964. For sale by the Superintendent of Documents. 